\title{
Glassy dynamics of electrons near the metal-insulator transition
}

\author{
Dragana Popović \\ National High Magnetic Field Laboratory, Florida State University \\ Tallahassee, Florida 32310, USA
}

\section{OXFORD}

UNIVERSITY PRESS 



\section{Preface}

This review first describes the evidence that strongly suggests the existence of the metal-insulator transition (MIT) in a two-dimensional electron system in Si regardless of the amount of disorder. Extensive studies of the charge dynamics demonstrate that this transition is closely related to the glassy freezing of electrons as temperature $T \rightarrow 0$. Similarities to the behavior of three-dimensional materials raise the intriguing possibility that such correlated dynamics might be a universal feature of the MIT regardless of the dimensionality. 



\section{Contents}

0.1 Introduction 1

0.2 Metal-insulator transition in two dimensions

0.3 Glassy freezing of electrons in two dimensions 15

0.4 Summary 28

0.5 Discussion 29

0.6 Acknowledgments 33

References 34 


\subsection{Introduction}

The discovery of many novel materials over the last couple of decades has revived interest in the metal-insulator transition (MIT), one of the longstanding, fundamental problems of condensed matter physics. Many such materials, including manganites, diluted magnetic semiconductors and high-temperature superconductors, are created by doping an insulating host and thus find themselves close to a conductor-insulator transition. There is substantial evidence that, in many cases, strong electronic correlations (Mott localization) and disorder (Anderson localization) both play an important role in this regime (see Miranda and Dobrosavljević 2005 for review). In general, the competition between the Coulomb repulsion, which favors a uniform distribution of electrons (Wigner crystallization), and disorder, which favors a random one, leads to the frustration in the system. This means that electrons are unable to satisfy these requirements simultaneously, resulting in the absence of a unique ground state and the emergence of a large number of metastable states or minima ("valleys") in the free energy landscape. Metastable states, which correspond to different charge configurations, are separated by high barriers, leading to slow dynamics, divergence of the equilibration time, and breaking of ergodicity. Such a system is called a Coulomb glass. However, there are many other types of glassy systems. A common denominator in all of them is their complex or "rugged" energy landscape (Fig. 0.1). The most extensively studied and best known glasses in condensed matter physics are probably conventional spin glasses (Binder and Young, 1986), such as Cu:Mn. On the other hand, Coulomb glasses were first anticipated theoretically almost three decades ago (Davies et al., 1982: Grünewald et al., 1982; Pollak and Ortuño, 1982; Davies et al., 1984; Pollak, 1984) in situations where electrons are strongly localized due to disorder, but experimental studies have been scarce. Some of the recent work on electron glasses in such strongly localized regime, away from the MIT, has been reviewed by Amir et al. (2011). In the opposite limit of well-delocalized electrons, one expects a single, well-defined ground state and the absence of glassiness. Not surprisingly, it is the behavior in the intermediate region, near the MIT, that has been most difficult to understand.

In the presence of disorder, the local electron density undergoes strong spatial fluctuations. Therefore, it is plausible to expect that, in the vicinity of the MIT, the local density in some areas may be higher than the average and correspond to the metallic state, whereas in the remaining regions, the local density may be lower than the average and the electrons are localized. In fact, it has been proposed that, in weakly doped Mott insulators near the MIT, the system will settle for a nanoscale phase separation 11 between a conductor and an insulator (Gor'kov and Sokol, 1987, Kivelson et al., 2003, Dagotto, 2002). New powerful experimental techniques, including imaging by scanning tunneling microscopy (Kohsaka et al., 2007), have indeed firmly established the existence of charge inhomogeneities in strongly correlated electron systems, such as cuprates (Orenstein and Millis, 2000; Dagotto, 2005). This obviously leads to the possibility for a myriad of competing charge configurations and the emergence of the associated glassy dynamics. Recent studies (Raičević et al., 2007.

\footnotetext{
${ }^{1}$ Global phase separation is not possible as it would violate charge neutrality.
} 


\section{Contents}

Raičević et al., 2008, Jelbert et al., 2008, Raičević et al., 2011) of a lightly doped, insulating $\mathrm{La}_{2-x} \mathrm{Sr}_{x} \mathrm{CuO}_{4}, x=0.03$, have found several clear signatures of glassy charge dynamics as temperature $T \rightarrow 0$, consistent with an underlying glassy ground state that results from Coulomb interactions. Further work is needed, however, to see how this glassy state evolves as the system approaches the transition from an insulator to a conductor.

It is also desirable to extend studies of charge dynamics to other types of materials to explore a possible generality of glassy freezing in strongly correlated systems near the MIT (Dobrosavljević et al., 2003: Pankov and Dobrosavljević, 2005: Miranda and Dobrosavljević, 2005). Many materials do exhibit complex behavior due to the existence of several competing ground states (Dagotto, 2005). In fact, the frustration caused by the competition of interactions on different length scales may give rise to glassy dynamics even in the absence of disorder (Schmalian and Wolynes, 2000), while even a small amount of disorder may favor glassiness over various static charge-ordered states (Pankov and Dobrosavljević, 2005). Even though the emergence of glassiness thus appears to be ubiquitous at low temperatures, Coulomb glasses and out-of-equilibrium systems in general remain poorly understood. Experimentally, studies of charge dynamics near the MIT in many materials are often complicated by the accompanying changes in magnetic or structural symmetry, as well as by the glassy freezing of spins. Doped semiconductors, such as Si:P and Si:B, are free from such complications. They have been used extensively to study the critical behavior near the MIT (Miranda and Dobrosavljević, 2005, Sarachik, 1995) but, in spite of some early hints of glassiness in the insulating regime (Paalanen et al., 1983: Monroe et al., 1987; Monroe, 1990), charge dynamics was not studied further until recently (Kar et al., 2003. Thorsmølle and Armitage, 2010), as discussed below.

Two-dimensional electron systems (2DES) in semiconductors (Ando et al., 1982), such as $\mathrm{Si}$, provide another relatively simple system for exploring the interplay of electronic correlations and disorder (for a review of disordered electronic systems, see

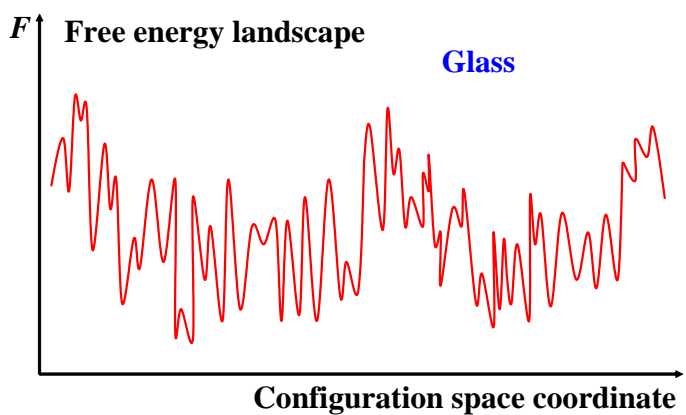

Fig. 0.1 The free-energy $(F)$ landscape of a glassy system. The horizontal axis represents the one-dimensional projection of the configurational coordinates of the degrees of freedom. 
Lee and Ramakrishnan 1985). They have an additional advantage that all the relevant parameters, carrier concentration, disorder and interactions, can be varied relatively easily. This review will describe some of the work that has demonstrated that the 2DES in $\mathrm{Si}$ is an excellent model system not only for studying the MIT in two dimensions (2D), but also for investigating the nearly universal nonequilibrium behavior exhibited by a large class of both three-dimensional (3D) and 2D systems (e.g. spin glasses, supercooled liquids, granular films). In fact, the work on the 2DES in Si provides additional strong evidence that many such universal features are robust manifestations of glassiness, regardless of the dimensionality of the system.

The dimensionality plays an important role in the MIT. In 2D, for example, the very existence of the metal and the MIT had been questioned for many years. Recently, considerable experimental evidence has become available in favor of such a transition. Some of that work has been described in several review papers (e.g. Kravchenko and Sarachik 2004) with a focus on very clean (low-disordered) samples. This review will first extend that discussion to the cases of higher disorder in order to (a) demonstrate that the 2D MIT is observed in all 2DES in Si regardless of the amount of disorder, (b) point out important similarities to the MIT in 3D systems, and (c) set the stage for the discussion of glassy dynamics near the MIT. As summarized below, the 2DES in Si exhibits all the main manifestations of glassiness: slow, correlated dynamics; nonexponential relaxations; diverging equilibration time, as $T \rightarrow 0$; aging and memory. The results provide strong support to theoretical proposals describing the 2D MIT as the melting of a Coulomb glass (Dobrosavljević et al., 2003, Thakur and Neilson, 1996: Thakur and Neilson, 1999: Chakravarty et al., 1999| Pastor and Dobrosavljević, 1999 Dalidovich and Dobrosavljević, 2002). The review concludes by further comparison to other complex materials, both 2D and 3D, and by identifying some open directions for future research.

\subsection{Metal-insulator transition in two dimensions}

In 2D systems in semiconductor heterostructures, the electron density $n_{s}$ can be varied easily over two orders of magnitude simply by applying voltage $V_{g}$ to the so-called gate electrode. At low $n_{s}$, the 2DES is strongly correlated: $r_{s} \gg 1$, where $r_{s}=E_{C} / E_{F} \propto$

$n_{s}^{-1 / 2}\left(E_{C}\right.$ is the average Coulomb energy per electron and $E_{F}$ is the Fermi energy; see also Kravchenko and Sarachik 2004 for more details). At the same time, the electrons "feel" a random potential (disorder) caused by charged impurities that are located away from the 2DES. Some of the striking experimental evidence for the MIT that occurs in a variety of "clean", i.e. low-disordered 2D systems has been presented also in the chapter by S. V. Kravchenko (this volume).

This section reviews work that demonstrates the existence of the 2D MIT regardless of the amount or type of disorder. The disorder, however, does affect the values of $n_{c}$, the critical density for the MIT, and the precise form of the temperature dependence of conductivity $\sigma(T)$. For the sake of clarity, it is important to recall that a qualitative distinction between a metal and an insulator exists only at $T=0: \sigma(T=0) \neq 0$ in the metal and $\sigma(T=0)=0$ in the insulator. The experiments were performed on a 2DES in Si metal-oxide-semiconductor field-effect transistors (MOSFETs), where the low density regime can be reached more easily compared to other semiconductors 


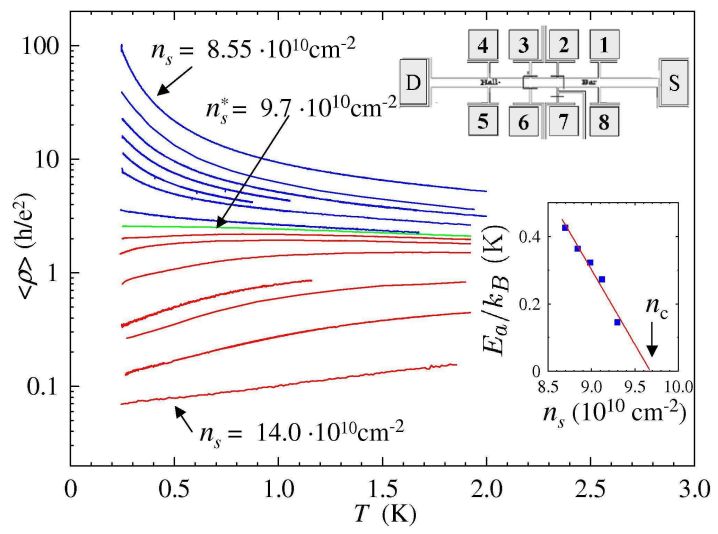

Fig. 0.2 High-mobility sample $\left(\mu \approx 2.5 \mathrm{~m}^{2} / \mathrm{Vs}\right)$ : resistivity $\rho=1 / \sigma$ vs. $T$ for $n_{s}\left(10^{10} \mathrm{~cm}^{-2}\right)=8.55,8.70,8.84,8.99,9.13,9.27,9.56,9.71,9.85,9.99,10.4,11.2,11.6,12.9$, 14.0 (from the top) (Jaroszyński et al., 2002b). Insets: a schematic of the sample (top view), and activation energies vs. $n_{s} ; n_{c} \approx n_{s}^{*}\left(\right.$ here $r_{s} \approx 17$ ).

(Kravchenko and Sarachik, 2004). In Si MOSFETs, the (Drude) mobility $\mu=\sigma /\left(n_{s} e\right)$ peaks as a function of $n_{s}$ because, at very high $n_{s}$ that are not of interest here, the scattering due to the roughness of the $\mathrm{Si}_{-} \mathrm{SiO}_{2}$ interface becomes increasingly important (Ando et al., 1982). The peak mobility at $4.2 \mathrm{~K}$ is commonly used as a rough measure of the amount of disorder.

\subsubsection{Effects of disorder}

High-mobility (low-disordered) samples. In high-mobility Si MOSFETs where, roughly speaking, the $4.2 \mathrm{~K}$ peak $\mu>2 \mathrm{~m}^{2} /(\mathrm{Vs})$, the low-temperature resistivity $\rho$ exhibits a strong, metallic drop $(d \rho / d T>0)$ with decreasing $T$ for $n_{s}>n_{s}^{*}$ (Fig. 0.2) (Kravchenko and Sarachik, 2004). $d \rho / d T$ changes sign at a density $n_{s}^{*}$ and becomes insulatinglike $(d \rho / d T<0)$ for $n_{s}<n_{s}^{*}$. Even though $d \rho / d T<0$ does not necessarily imply that $\rho$ diverges at zero temperature (i.e. that $\sigma(T=0)=0$ ), the density $n_{s}^{*}$ has been often assumed to represent the critical density for the MIT. However, other, more appropriate methods have been also used to identify $n_{c}$. For example, $n_{c}$ was determined based on both a vanishing activation energy and a vanishing nonlinearity of current-voltage characteristics when extrapolated from the insulating phase (Pudalov et al., 1993 Shashkin et al., 2001). It was established that $n_{c} \approx n_{s}^{*}$, although a small but systematic difference of a few percent has been reported such that $n_{c}<n_{s}^{*}$ (Pudalov et al., 1998a; Altshuler et al., 2001). Fig. 0.2 inset illustrates the use of activation energies, determined from the fits of the data at the lowest $n_{s}$ and $T$ to the simply activated form $22 \propto \exp \left(E_{A} / k_{B} T\right)$.

In the vicinity of $n_{c}\left(\right.$ or $\left.n_{s}^{*}\right)$, the resistivity curves can be collapsed onto the same scaling function of a single parameter $T_{0}$, i.e. $\rho\left(T, n_{s}\right)=\rho_{c} f\left(T / T_{0}\right)$ (Kravchenko et al., 1994).

${ }^{2}$ Close enough to $n_{c}$, the data can be fitted almost equally well to the variable-range hopping law. This gives the same values of $n_{c}$ within the error of the fit. 
$T_{0}$ is the same function of $\delta_{n} \equiv\left(n_{s}-n_{c}\right) / n_{c}$ on both the metallic and the insulating sides of the transition, $T_{0} \propto\left|\delta_{n}\right|^{z \nu}(z$ - dynamical exponent, $\nu$ - correlation length exponent), $z \nu=1.6$ (Kravchenko et al., 1995). Here $\rho_{c}\left(n_{c}\right)$ is the so-called "separatrix", the temperature independent critical resistivity curve. On the metallic side, the scaling does not work at higher $T$, in the regime where $\rho(T)$ goes through a maximum and then decreases as $T$ is raised further (Kravchenko and Sarachik, 2004). On general grounds, the scaling of $\rho\left(n_{s}, T\right)$ at low enough $T$ represents a strong indication for the existence of the metal-insulator transition at $T=0$ (Sachdev, 1999).

At somewhat higher $n_{s}$ in the metallic phase, it becomes apparent that, after the initial rapid drop, $\rho(T)$ becomes a very weak function as $T \rightarrow 0$ (Pudalov et al., 1998a Pudalov et al., 1998b). In fact, it is so weak that, on a logarithmic scale, it appears to saturate. As a result of this "saturation" of $\rho(T)$, single-parameter scaling fails at the lowest temperatures (Pudalov et al., 1998a). However, a careful analysis of the data shows (Kim et al., 1998, Washburn et al., 1999a; Washburn et al., 1999b) that all of the $\sigma\left(n_{s}, T\right)$ (or $\left.\rho\left(n_{s}, T\right)\right)$ curves can be scaled according to the more general form (Belitz and Kirkpatrick, 1994) $\sigma\left(n_{s}, T\right)=\sigma_{c}(T) f\left(T / T_{0}\right)$, where the critical conductivity $\sigma_{c}=\sigma\left(n_{c}, T\right) \propto T^{x}$, i.e. it vanishes as $T \rightarrow 0$. In other words, the critical conductivity is not the "separatrix", but instead it belongs to the insulating family of curves: $n_{c}<n_{s}^{*}$, consistent with other studies (Pudalov et al., 1998a; Altshuler et al., 2001). The exponent $x$, however, is very small: $x=0.1-0.2$ (Kim et al., 1998) Washburn et al., 1999a; Washburn et al., 1999b), so that a direct observation of $\sigma_{c}(T)$ is very difficult at experimentally accessible temperatures. A small value of $x$ is also the reason for the apparent success of the single-parameter scaling in the limited range of $T$ and $n_{s}$. It is important to note that scaling with $x \neq 0$, also observed in 3D materials near the MIT (Sarachik, 1995), does not contradict (Belitz and Kirkpatrick, 1994) any fundamental principle for 2D systems. Indeed, violations of "Wegner scaling" (Belitz and Kirkpatrick, 1994), where $x=(D-2) / z(D$ - dimensionality), were predicted for certain microscopic models (Castellani et al., 1987; Kirkpatrick and Belitz, 1994 Belitz and Kirkpatrick, 1995) with strong spin-dependent components of the Coulomb interactions.

While the general scaling form describes the data satisfactorily as $T \rightarrow 0$, it would be desirable to explore scaling and the precise form of $\sigma_{c}(T)$ over a wider range of $T$. It turns out that the situation becomes simpler in samples with more disorder, where the onset of "saturation" of $\sigma$ (or $\rho$ ) in the metallic phase is pushed to higher $T$.

Intermediate-mobility samples and effects of local magnetic moments. Although the metallic temperature dependence of conductivity, $d \sigma / d T<0$ (i.e. $d \rho / d T>0$ ), is less pronounced in samples with moderate mobility $\left(4.2 \mathrm{~K}\right.$ peak $\left.\mu \sim 1 \mathrm{~m}^{2} / \mathrm{Vs}\right)$, it was demonstrated early on (Popović et al., 1997) that scaling $\sigma\left(n_{s}, T\right)=\sigma_{c} f\left(T / T_{0}\right)$ is obeyed with exactly the same value of the exponent $z \nu=1.6$ as in high-mobility samples. While the value of $n_{c}$, defined as $n_{s}^{*}$, was higher than in high-mobility devices, the corresponding $r_{s} \approx 13$ was still comparably large.

\footnotetext{
${ }^{3}$ A study of Si MOSFETs with different peak mobilities indicated a systematic increase of $n_{c}$ with disorder; $n_{c}$ was defined as the "separatrix" $n_{s}^{*}$ (Pudalov et al., 1998a).
} 

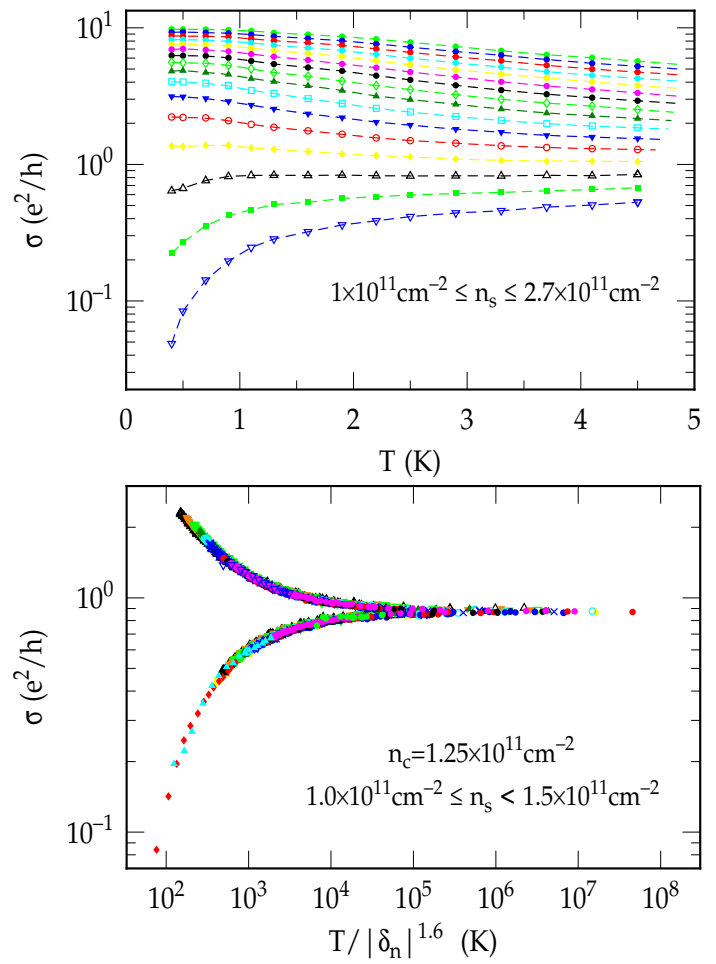

Fig. 0.3 Intermediate-mobility sample $\left(\mu \approx 1 \mathrm{~m}^{2} / \mathrm{Vs}\right.$; sample 12$)$ : conductivity $\sigma$ vs. $T$ for different $n_{s}$ (top), and scaling of $\sigma$ with temperature for the same data (and other $n_{s}$ not shown) in the $n_{s}$ range given on the plot (bottom). $\delta_{n} \equiv\left(n_{s}-n_{c}\right) / n_{c}$ is the reduced density; $n_{c} \approx n_{s}^{*}$ (the corresponding $r_{s} \approx 15$ ). For $n_{s} \geq n_{s}^{*}$, the scaling works only for $T \geq 1.1 \mathrm{~K}$. The substrate (back-gate) bias on the sample was $V_{\text {sub }}=-40 \mathrm{~V}$ (Feng et al., 1999).

By extending the measurements to lower $T$ (Feng et al., 1999), however, the "saturation" of $\sigma(T)$ was observed to take place in the metallic phase at $T$ as high as $\sim 1 \mathrm{~K}$ (Fig. 0.3 top). Moreover, the separatrix acquired an insulatinglike $(d \sigma / d T>0)$ temperature dependence. As a result, the simple, single-parameter scaling around the separatrix works only at $T>1.1 \mathrm{~K}$ on the metallic side (Fig. 0.3 bottom). Thus the data are qualitatively similar to those on high-mobility samples, indicating that $n_{c}<n_{s}^{*}$. However, since $\sigma(T)$ in the metallic phase $\left(n_{s}>n_{c}\right)$ does not have a simple enough form over a sufficiently wide range of $T$, it is still difficult to make reliable extrapolations to $T=0$. Fortunately, in Si MOSFETs it is also possible to change the type of the disorder in the same sample. As shown below, this results in a surprisingly simple, precise form of $\sigma(T)$ over a very wide range of $T$, allowing reliable zero-temperature extrapolations and striking scaling behavior.

For a $2 \mathrm{DES}$ in $\mathrm{Si}$, the disorder is due to the oxide charge scattering (scattering by ionized impurities randomly distributed in $\mathrm{SiO}_{2}$ within a few $\AA$ of the interface) and to the roughness of the $\mathrm{Si}_{-} \mathrm{SiO}_{2}$ interface (Ando et al., 1982). For a fixed $n_{s}$, it is possible to change the disorder by applying bias $V_{s u b}$ to the Si substrate (back gate). In 
particular, the reverse (negative) $V_{\text {sub }}$ moves the electrons closer to the interface, which increases the disorder. It also increases the splitting between the subbands since the width of the triangular potential well at the interface is reduced by applying negative $V_{\text {sub }}$. Usually, only the lowest subband is occupied at low $T$, giving rise to the $2 \mathrm{D}$ behavior. In sufficiently disordered samples, however, the band tails associated with the upper subbands can be so long that some of their strongly localized states may be populated even at low $n_{s}$, and act as additional scattering centers for $2 \mathrm{D}$ electrons. In particular, since at least some of them must be singly populated due to a large on-site Coulomb repulsion (tens of meV), they may act as local magnetic moments. Clearly, the negative $V_{\text {sub }}$ reduces this type of scattering by depopulating the upper subband.

Therefore, by varying $V_{s u b}$, it is possible to study the effect of local magnetic moments on the transport properties of the conduction electrons in a systematic and controlled way. It has been established (Feng et al., 1999) that scattering by local magnetic moments suppresses the metallic behavior with $d \sigma / d T<0$. Indeed, the data suggest that in the $T \rightarrow 0$ limit, the $d \sigma / d T<0$ (i. e. $d \rho / d T>0$ ) behavior is suppressed by an arbitrarily small amount of scattering of the conduction electrons by disorder-induced local moments (Feng et al., 1999). Figures 0.3(top) and 0.4(a) illustrate the striking effect of local moments on $\sigma(T)$ in the same sample: while the "usual", metallic behavior with $d \sigma / d T<0$ is observed in the absence of local moments [Fig. [0.3 top], $\sigma(T)$ curves become insulatinglike $(d \sigma / d T>0)$ for all $n_{s}$ after many local moments are introduced with $V_{\text {sub }}$ [Fig. [0.4(a)]. However, this does not necessarily indicate the destruction of the metallic phase. In disordered 3D metals, for example, it is well known that the derivative $d \sigma / d T$ can be either negative or positive near the MIT (Lee and Ramakrishnan, 1985). On the other hand, the metallic behavior where $\sigma$ decreases but does not go to zero (as expected for an insulator) when $T \rightarrow 0$ is new and unexpected in $2 \mathrm{D}$.

The analysis of the insulatinglike $\sigma(T)$ curves in Fig.0.4(a) shows (Feng et al., 2001) that they follow a very simple and precise form over a broad (two decades) range of $T$ and $n_{s}: \sigma\left(n_{s}, T\right)=\sigma\left(n_{s}, T=0\right)+A\left(n_{s}\right) T^{2}$ [Fig. 0.4(b)]. The high quality of the fits allows a reliable extrapolation of $\sigma\left(n_{s}, T=0\right)$, whose finite (i. e. nonzero) values mean that, in spite of the decrease of $\sigma\left(n_{s}, T\right)$ with decreasing $T$, the $2 \mathrm{D}$ system is in the metallic state. In particular, the zero-temperature conductivity $\sigma\left(n_{s}, T=0\right)$ is a power law function of $\delta_{n}$ [Fig. 0.4(c)]: $\sigma\left(n_{s}, T=0\right) \propto \delta_{n}^{\mu}(\mu \approx 3)$, as expected in the vicinity of a quantum critical point (Goldenfeld, 1992), such as the MIT. The power law holds over a very wide range of $\delta_{n}$ (up to 5) similar to what has been observed (Rosenbaum et al., 1980) in Si:P near the MIT. In addition, even though the MIT occurs at different $n_{c}$ in different samples, the critical exponents $\mu$ are the same [Fig. 0.4(c)], as expected from general arguments (Goldenfeld, 1992). It has been also demonstrated (Feng et al., 2001) that, near the MIT, the data obey dynamical scaling $\sigma\left(n_{s}, T\right)=\sigma_{c}(T) f\left(T / \delta_{n}^{z \nu}\right)$ [Fig. 0.4(d)] with a temperature dependent critical conductivity $\sigma_{c}=\sigma\left(n_{s}=n_{c}, T\right) \propto T^{x}(z \nu=1.3 \pm 0.1, x \approx 2.6$, $\mu=x(z \nu)=3.4 \pm 0.4)$, both in agreement with theoretical expectations near a quantum phase transition (Belitz and Kirkpatrick, 1994) and consistent with the extrapolations of $\sigma(T)$ to $T=0$. 

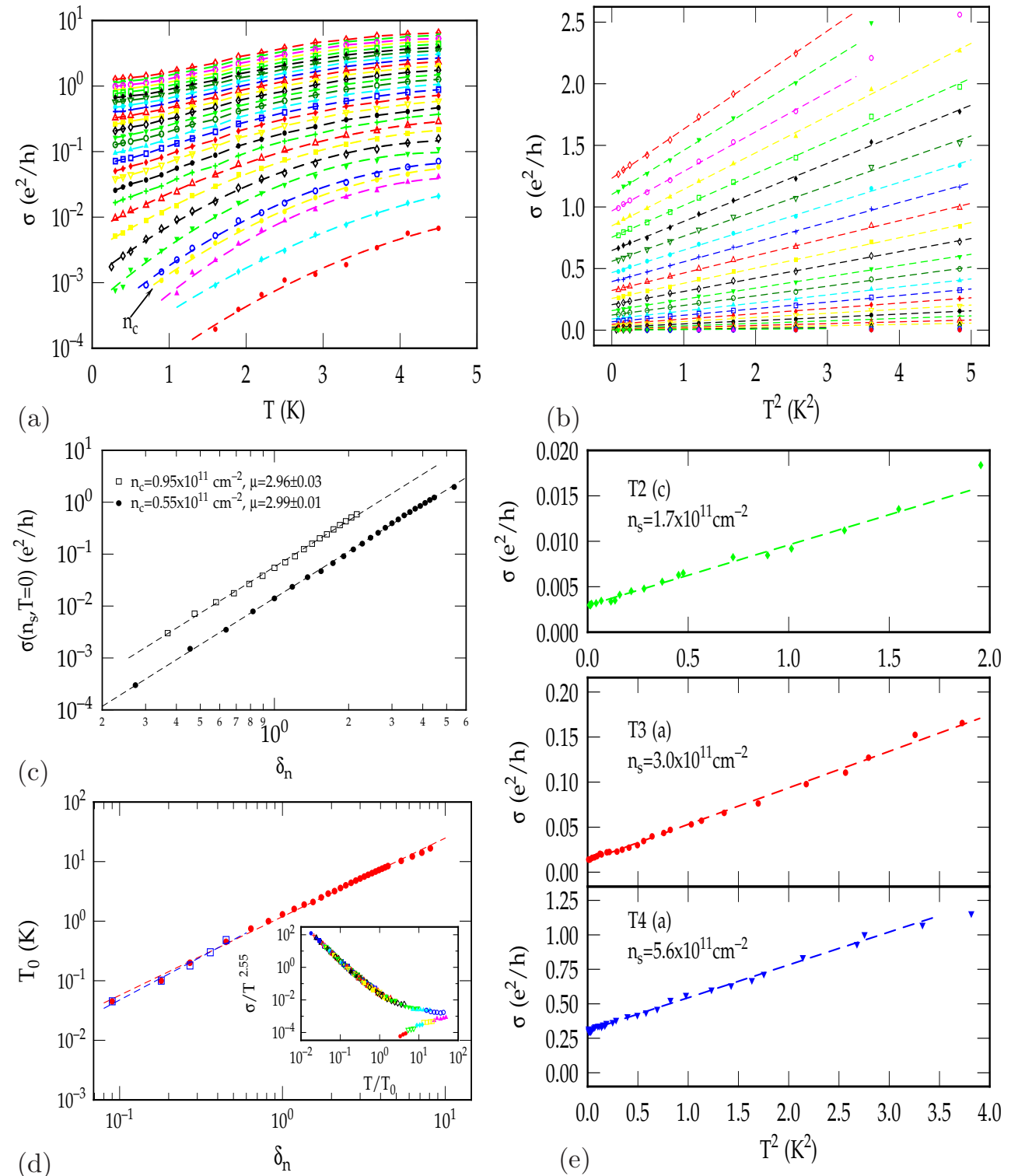

Fig. 0.4 (a)-(d) Adapted from Feng et al. (2001); the same intermediate-mobility sample (sample 12) as in Fig. 0.3 but here $V_{\text {sub }}=+1 \mathrm{~V}$. (a) $\sigma(T)$ for $0.3 \leq n_{s}\left(10^{11} \mathrm{~cm}^{-2}\right) \leq 3.0$ (bottom to top) in steps of $0.1 \times 10^{11} \mathrm{~cm}^{-2}$. (b) The same $\sigma(T)$ data plotted vs. $T^{2}$; the lowest $n_{s}=0.7 \times 10^{11} \mathrm{~cm}^{-2}$ and $0.3 \leq T \leq 2.2 \mathrm{~K}$. Other measurements show that this $\sigma(T)$ holds at least down to $0.020 \mathrm{~K}$ (Feng et al., 2001, Eng et al., 2002). (c) $\sigma\left(n_{s}, T=0\right) v s . \delta_{n}$ for samples 12 (dots) and 9 (squares). The dashed lines are fits with the slopes equal to the critical exponent $\mu$. At the MIT, the corresponding $r_{s} \approx 22$ and 17 for the two samples, respectively. (d) Scaling parameter $T_{0}$ as a function of $\left|\delta_{n}\right|$ for sample 12; open symbols: $n_{s}<n_{c}$, closed symbols: $n_{s}>n_{c}$. The dashed lines are fits with slopes $1.4 \pm 0.1$ and $1.32 \pm 0.01$, respectively. Inset: scaling of raw data $\sigma / \sigma_{c} \sim \sigma / T^{x}$ in units of $e^{2} / h \mathrm{~K}^{2.55}$ for all $n_{s}$ shown in (a) and $T<2$ K. (e) Adapted from Sjöstrand and Stiles (1975) and Sjöstrand et al. (1976): $\sigma(T)$ vs. $T^{2}$ for three different samples and densities. The $4.2 \mathrm{~K}$ peak mobility was between 0.15 and $0.33 \mathrm{~m}^{2} / \mathrm{Vs}$. 
The $T^{2}$ form of $\sigma(T)$ is well established for metals containing local magnetic moments, and it is believed to result from the Kondo effect (Hewson, 1993). In fact, there is no other known mechanism that results in an increase of $\sigma$ as $T^{2}$. Here this feature provides the most direct evidence of the presence of local magnetic moments. It should be noted that, in general, one expects the $T^{2}$ behavior for a quantum impurity embedded in a Fermi liquid in any dimension. While the nature of this novel metallic state in 2D may require further study, its simple $\sigma(T)$ allows for an unambiguous extrapolation to $T=0$. The zero-temperature conductivity $\sigma\left(n_{s}, T=0\right)$ decreases continuously, and follows a distinct power-law behavior as the MIT is approached. In particular, metallic $\sigma$ as small as $10^{-3} e^{2} / h$ has been observed (Feng et al., 2001), in a striking contrast to anything that has been reported in other 2D systems when $d \sigma / d T<0$. A similar observation in 3D systems (Rosenbaum et al., 1980) has demonstrated the absence of minimum metallic conductivity, and has had a profound impact on shaping the theoretical ideas about the MIT.

2DES in Si MOSFETs have been studied extensively for more more than four decades, so it is interesting that the $T^{2}$ behavior has been identified only relatively recently (Feng et al., 2001). A thorough examination of the early literature reveals, however, that the samples discussed here are representative of a broad class of Si MOSFETs historically (and somewhat unfairly) known as "nonideal" samples (Ando et al., 1982). "Nonideal" samples could be made more "ideal" by applying $V_{\text {sub }}$ and vice versa (Ando et al., 1982), consistent with recent studies (Feng et al., 1999: Feng et al., 2001) discussed above. Figure 0.4(e) shows an example of $\sigma(T)$ measured on samples with a modest peak mobility between 0.15 and $0.33 \mathrm{~m}^{2} / \mathrm{Vs}$ (Sjöstrand and Stiles, 1975 , Sjöstrand et al., 1976). The "saturation" (on a log scale) of $\sigma(T)$ observed for $T<1 \mathrm{~K}$ was puzzling at the time, but the replotted data show clear $T^{2}$ behavior.

Low-mobility (highly disordered) samples. Samples with very low $4.2 \mathrm{~K}$ peak mobility $\left(\mu<0.1 \mathrm{~m}^{2} / \mathrm{Vs}\right)$ have attracted less attention because they do not exhibit a pronounced, if any, $d \sigma / d T<0$ metallic behavior. However, they not only exhibit the 2D MIT, but also other similarities to the behavior of "clean" 2DES, such as the onset of glassy charge dynamics (Section 0.3). In some ways, it is even advantageous to investigate samples with a lot of disorder. For example, the relevant electron densities, such as $n_{c}$, are pushed to higher values (higher $E_{F}$ ), so that it is possible to reach much lower effective temperatures $T / T_{F}\left(T_{F}-\right.$ Fermi temperature) than in high-mobility samples. Therefore, comparative studies of samples with varying amounts of disorder should provide valuable insights into the physics of systems near the MIT.

Detailed studies of transport and electron dynamics near the MIT have been performed on a set of Si MOSFETs with the $4.2 \mathrm{~K}$ peak mobility of only $0.06 \mathrm{~m}^{2} / \mathrm{Vs}$ with the applied $V_{s u b}=-2 \mathrm{~V}$ (Bogdanovich and Popović, 2002b). This value of $V_{\text {sub }}$ maximizes the peak mobility by removing the contribution of scattering by local magnetic moments (see Sec. 0.2.1 above), at least in the experimental $T$-range. Because of the glassy fluctuations of $\sigma$ with time $t$ at low $n_{s}$ and $T$ (see Sec. 0.3), the carrier density had to be changed at relatively high $T$ (here $\sim 0.8 \mathrm{~K}$ ) in small steps in order to obtain reproducible values of the time-averaged conductivity $\langle\sigma\rangle$. The behavior of $\left\langle\sigma\left(n_{s}, T\right)\right\rangle$ (Fig. 0.5 left) turns out to be quite similar to that of high-mobility $\mathrm{Si}$ MOSFETs. At the highest $n_{s}$, for example, the devices exhibit metallic behavior with 
10 Contents

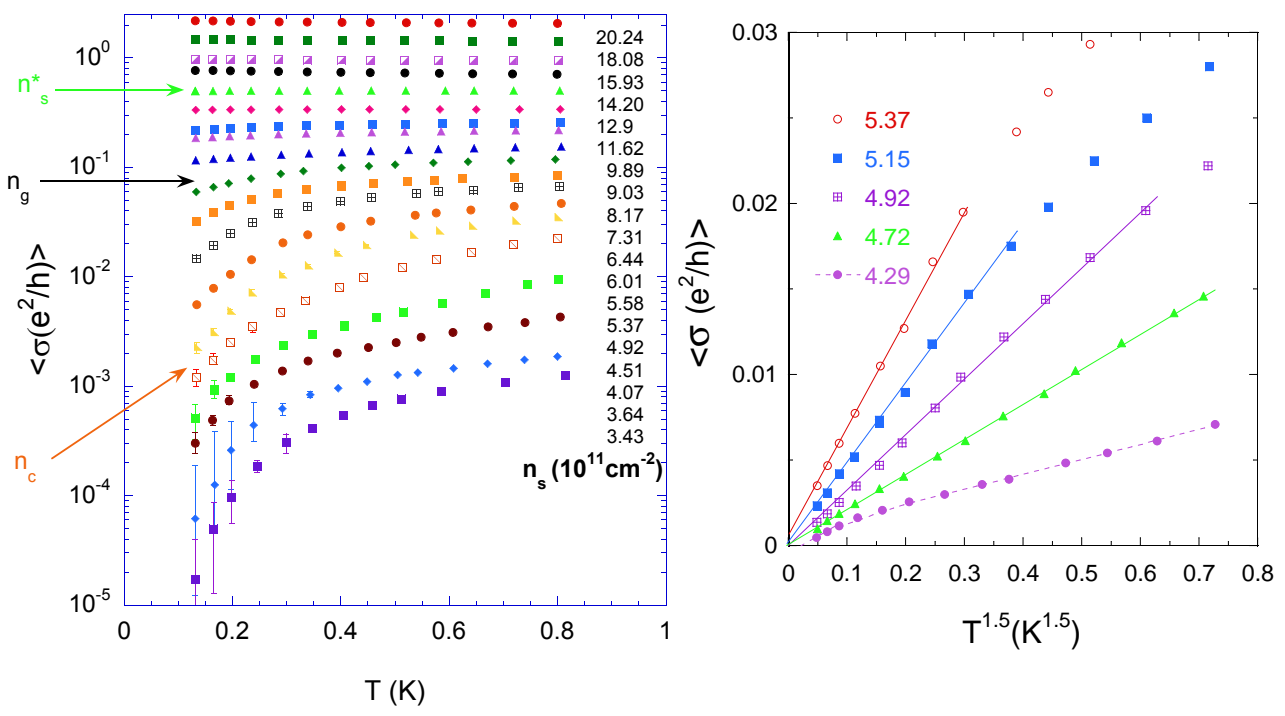

Fig. 0.5 Low-mobility sample $\left(\mu \approx 0.06 \mathrm{~m}^{2} / \mathrm{Vs}\right)$ (Bogdanovich and Popović, 2002b). Left: time-averaged $\langle\sigma\rangle$ vs. $T$ for different $n_{s}$. The error bars show the size of the fluctuations with time. $n_{s}^{*}, n_{g}$, and $n_{c}$ are marked by arrows $\left(n_{g}-\right.$ glass transition density). Right: $\langle\sigma\rangle$ vs. $T^{1.5}$ for a few $n_{s}\left(10^{11} \mathrm{~cm}^{-2}\right)$ near $n_{c}$. The solid lines are fits; the dashed line is a guide to the eye, clearly showing insulating behavior $[\langle\sigma(T \rightarrow 0)\rangle=0]$.

$d\langle\sigma\rangle / d T<0$. The change of $\langle\sigma\rangle$ in a given $T$ range, however, is small (only $6 \%$ for the highest $n_{s}=20.2 \times 10^{11} \mathrm{~cm}^{-2}$ ) as observed in other Si MOSFETs with a large amount of disorder (Pudalov et al., 1998a). Even though the density $n_{s}^{*}=12.9 \times 10^{11} \mathrm{~cm}^{-2}$ at the separatrix (Bogdanovich and Popović, 2002b: Bogdanovich and Popović, 2002a) is much higher than in "clean" samples, the value of $\left\langle\sigma\left(n_{s}^{*}\right)\right\rangle=0.5 e^{2} / h$ is similar, which, according to Drude formula, corresponds to $k_{F} l \lesssim 1\left(k_{F}\right.$ - Fermi wave vector, $l-$ mean free path). Likewise, at the lowest $n_{s}$, the data are best described by the simply activated form $\langle\sigma\rangle \propto \exp \left(-E_{A} / k_{B} T\right)$.

Perhaps the most striking difference between high- and low-mobility samples first becomes apparent when the vanishing of activation energy $E_{A}$ is used to determine $n_{c}$. While in "clean" samples this gives $n_{c} \lesssim n_{s}^{*}$ (Sec. 0.2.1), here $E_{A}$ vanishes at $n_{c} \approx 5 \times 10^{11} \mathrm{~cm}^{-2}$, which is more than a factor of two smaller than $n_{s}^{*}$ (Fig. 0.5 left). This suggests that there is a wide range of $n_{s}$ on the metallic side of the MIT where $\sigma(T)$ is insulatinglike. Indeed, close to $n_{c}$, the best phenomenological fit to the data is the metallic power-law behavior $\left\langle\sigma\left(n_{s}, T\right)\right\rangle=a\left(n_{s}\right)+b\left(n_{s}\right) T^{x}$ with $x \approx 1.5$ (Fig. 0.5 right) (Bogdanovich and Popović, 2002b). The fitting parameter $a\left(n_{s}\right)$ is relatively small and, in fact, vanishes for $n_{s}\left(10^{11} \mathrm{~cm}^{-2}\right)=4.72$ and 4.92 . Therefore, $n_{c}=(5.0 \pm 0.3) \times 10^{11} \mathrm{~cm}^{-2}\left(r_{s} \sim 7\right)$ based on the data on both metallic and insulating sides of the MIT. Of course, a simple power law $\left\langle\sigma\left(n_{c}, T\right)\right\rangle \propto T^{x}$ is consistent not only with general expectations near the MIT and the behavior observed in 3D systems (Belitz and Kirkpatrick, 1994), but also with the careful analysis of high-mobility 2DES (Kim et al., 1998; Washburn et al., 1999a; Washburn et al., 1999b) (Sec. 0.2.1) 
and those with local magnetic moments (Feng et al., 2001) (Sec.0.2.1). Here the exponent $x$ takes a distinctly different value, presumably reflecting the different universality classes of those situations.

The surprising non-Fermi liquid $T^{3 / 2}$ behavior is consistent with theory (Dalidovich and Dobrosavljević, 2002 for the transition region between a Fermi liquid and an (insulating) electron glass. Indeed (see Sec. 0.3), the transition into a charge (Coulomb) glass in low-mobility samples takes place as $T \rightarrow 0$ at a density $n_{g}$, such that $n_{c}<n_{g}<n_{s}^{*}$ (Fig. 0.5 left). The $T^{3 / 2}$ correction is characteristic of transport in the intermediate, $n_{c}<n_{s}<n_{g}$ region where the dynamics is glassy, but where $\sigma$ is still metallic $[\sigma(T \rightarrow 0) \neq 0]$ albeit so small that $k_{F} l<1$. Such "bad metals" include a variety of strongly correlated materials with unusual properties (Emery and Kivelson, 1995). Interestingly, the $T^{3 / 2}$ behavior can be revealed also in high-mobility 2DES by applying a parallel magnetic field.

\subsubsection{D metal-insulator transition in a parallel magnetic field}

Since magnetic field $B$ applied parallel to the 2DES plane couples only to electrons' spins, it is often used to probe the importance of spin, as opposed to charge, degrees of freedom. Some of the intriguing results that have been obtained in parallel $B$ in the vicinity of the zero-field 2D MIT have been described in the review by Kravchenko and Sarachik (2004). One of the main issues has been the fate of the metallic phase in a parallel $B$. From the insulating side, the critical density $n_{c}(B)$ can be determined by extrapolating to zero the activation energy and nonlinearity of current-voltage characteristics (Sec. 0.2.1). For $n_{s}>n_{c}(B)$, however, the metallic $d \sigma / d T<0$ behavior observed in high-mobility samples is suppressed by $B$, making it even more difficult to determine the critical density from the metallic side. Nevertheless, a careful analysis reveals exactly the same metallic $T^{3 / 2}$ correction in high-mobility samples in parallel magnetic fields as in highly disordered samples in zero magnetic field, confirming the existence of the MIT in those two cases.

Figure 0.6(a) shows the $\left(n_{s}, B, T=0\right)$ phase diagram obtained for a high-mobility sample (Jaroszyński et al., 2004b). The critical densities $n_{c}(B)$ were first determined using the activation energy method. Good agreement was found with the $n_{c}(B)$ dependence obtained on very similar samples using both activation energies and nonlinear current-voltage characteristic: 4 (Shashkin et al., 2001). At low fields, $n_{c}$ increases with $B$, and then it saturates for $B \gtrsim 4 \mathrm{~T}$, consistent with other studies that show that the 2DES is here fully spin polarized (Okamoto et al., 1999: Vitkalov et al., 2000. Tutuc et al., 2001). Even though $\sigma(T)$ is very weak at higher $n_{s}$, it is interesting to attempt to determine the separatrix, where $d \sigma / d T=0$. The corresponding density $n_{s}^{*}(B)>n_{c}(B)$ and, most intriguingly, within the measurement error, it coincides with $n_{g}(B)$, the glass transition density (Jaroszyński et al., 2004a). $n_{g}(B)$ was determined independently, based on the measurements of the fluctuations in $\sigma(t)$ (Jaroszyński et al., 2004b) (Sec. 0.3.1).

The important question to address is the form of $\sigma(T)$ in the narrow $n_{c}(B)<$ $n_{s}<n_{g}(B)$ region. Here the data are best described by the metallic $(\langle\sigma(T=0)\rangle \neq 0)$

${ }^{4}$ Because of the small sample to sample differences in the amount of disorder, the data from Shashkin et al. 2001 have been shifted up by $0.85 \times 10^{10} \mathrm{~cm}^{-2}$ to make the $n_{c}(B=0)$ values coincide. 


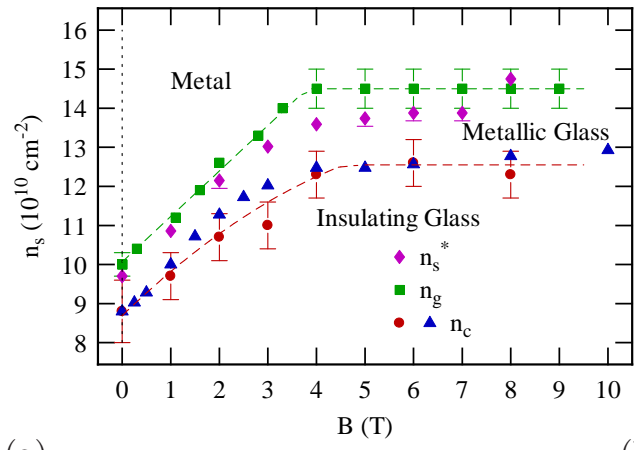

(a)

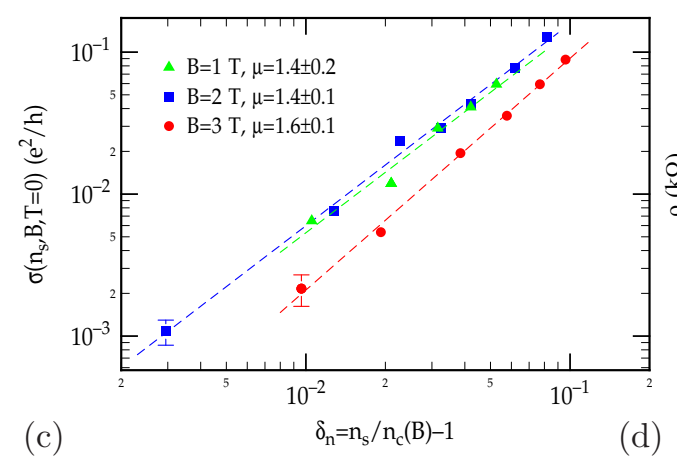

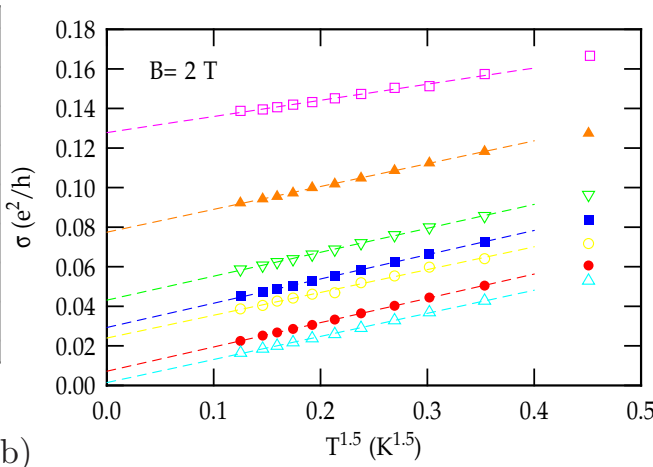

(b)

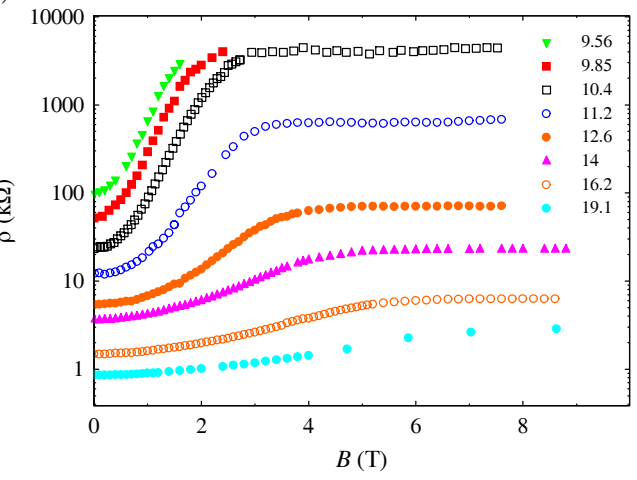

Fig. 0.6 High-mobility Si MOSFET. (a) $T=0$ phase diagram in a parallel magnetic field (Jaroszyński et al., 2004b). The dashed lines guide the eye. The $n_{c}$ values are from Jaroszyński et al. (2004b) (dots) and Shashkin et al. (2001) (triangles). The glass transition takes place at $n_{g}(B)>n_{c}(B)$, giving rise to an intermediate, metallic glass phase. The density at the separatrix $n_{s}^{*} \approx n_{g}$ within the error for all $B$. (b) $\sigma$ vs. $T^{1.5}$ for several $n_{s}$ in the metallic glass phase for $B=2 \mathrm{~T}\left(n_{s}\left(10^{10} \mathrm{~cm}^{-2}\right)=11.9,11.6,11.3,11.2,11.0,10.9,10.7\right.$ from top; $\left.n_{c}(B=2 \mathrm{~T})=10.67 \times 10^{10} \mathrm{~cm}^{-2}\right)$ for the same sample. Dashed lines are fits. (c) Zero-temperature conductivity $\sigma(T=0) \propto \delta_{n}^{\mu}$ obtained from the fits shown in (b). (d) The positive magnetoresistance for the same sample (Jaroszyński et al., 2004b) and different densities $n_{s}\left(10^{10} \mathrm{~cm}^{-2}\right)$, as shown. A strong increase in MR reflects a magnetic-field driven MIT at $n_{c}(B)$.

power-law form $\left\langle\sigma\left(n_{s}, B, T\right)\right\rangle=\left\langle\sigma\left(n_{s}, B, T=0\right)\right\rangle+b\left(n_{s}, B\right) T^{1.5}$ [Fig. 0.6(b)], similar to what was observed in the metallic glassy phase of highly disordered samples at $B=0$ (Sec. 0.2.1). The extrapolated $T=0$ conductivities go to zero precisely at $n_{c}(B)$, in a power-law fashion $\left\langle\sigma\left(n_{s}, B, T=0\right)\right\rangle \propto \delta_{n}^{\mu}$ with $\mu \approx 1.5$ [Fig. [0.6(c); $\delta_{n}=n_{s} / n_{c}(B)-1$ ] (Jaroszyński et al., 2004b) that is in agreement with theoretical expectations near a quantum phase transition (Belitz and Kirkpatrick, 1994). Interestingly, there is some evidence (Fletcher et al., 2001) of similar behavior at $B=0$ with $\mu \sim 1-1.5$, obtained by extrapolating to $T=0$ the "saturation" of $\sigma(T)$ in the $d \sigma / d T<0$ regime of different high-mobility Si MOSFETs. The striking power-law behavior shown in Fig. 0.6(c) and 
the remarkable agreement between $n_{c}(B)$ obtained from $\sigma(T)$ on both insulating and metallic sides of the MIT are strong evidence for the survival of the MIT and the metallic phase in parallel $B$.

In low-disordered ("clean") samples at $B=0$, the critical density $n_{c} \lesssim n_{s}^{*} \approx n_{g}$ [Sec. 0.2.1] Fig. [0.6(a)] and the intermediate, metallic glass phase is practically absent. A parallel $B$, however, increases its width, allowing the emergence of the characteristic $T^{3 / 2}$ correction to $\sigma$. The increase of both $n_{g}$ and $n_{c}$, and the broadening of the metallic glass phase with $B$ can be understood to result from the suppression of screening by a parallel $B$ (Dolgopolov and Gold, 2000 Herbut, 2001), which increases the effective disorder. This, in turn, favors glassiness, consistent with theoretical expectations (Dobrosavljević et al., 2003), and makes the behavior of "clean" samples more similar to that of highly disordered ones (Sec. 0.2.1). The existence of the glass transition in high parallel $B$, where the 2DES is spin polarized, provides evidence that charge, not spin, degrees of freedom are responsible for glassy ordering. This result clearly imposes a strong constraint on the types of theories that can be formulated to describe this phenomenon. Likewise, the broadening of the metallic glass phase with $B$ also indicates that its existence is not due to spin.

For $n_{s}$ near $n_{c}(B=0)$, 2DES in various semiconductors exhibit a strong, positive magnetoresistance (MR) (Kravchenko and Sarachik, 2004), which has been a subject of great interest. The MR saturates at the field that corresponds to the full spin polarization (Okamoto et al., 1999, Vitkalov et al., 2000 Tutuc et al., 2001 Vitkalov et al., 2001). As shown in Fig. [0.6(d) for the same high-mobility Si MOSFET discussed above, the MR jump is strong only for $n_{s}$ not too far from $n_{c}(B=0)$ since it takes place as the system undergoes a magnetic-field driven MIT at $n_{c}(B)$ [Fig. 0.6(a)]. In the metallic phase where $n_{s} \gg n_{c}(B>4 \mathrm{~T})$, the MR is weak.

In low-mobility Si MOSFETs, where the metallic glass phase is clearly observable already at $B=0$, the $\left(n_{s}, B, T=0\right)$ phase diagram has not been studied. However, it is plausible to expect that the parallel $B$ will broaden the metallic glass phase even further.

Finally, in intermediate-mobility 2DES with local magnetic moments, it is relatively easy to map out the $\left(n_{s}, B, T=0\right)$ phase diagram because of the simple form of $\sigma(T)$ that holds over a wide range of $T$. In parallel $B, \sigma\left(n_{s}, T\right)$ data (Fig. 0.7 top) are qualitatively similar to the $B=0$ case (Sec. 0.2.1) (Eng et al., 2002). At the lowest $n_{s}<n_{c}$, for example, $\sigma$ decreases exponentially with decreasing $T$, indicating an insulating state at $T=0$. For $n_{s}>n_{c}, \sigma(T)$ is weaker and its curvature is the opposite from the one expected for an insulating state. It clearly extrapolates to a finite value as $T \rightarrow 0$, indicating a metallic phase (Sec. 0.2.1). $n_{c}$ is identified as the density where $\sigma_{c}=\sigma\left(n_{s}=n_{c}, T\right) \propto T^{x}$ (Fig. 0.7 top), consistent with the $B=0$ case and in agreement with general arguments (Belitz and Kirkpatrick, 1994). The exponent $x=2.7 \pm 0.4$ remains constant as a function of $B$. The critical density $n_{c}$ determined in this way at each given $B$ allows the mapping of the $\left(n_{s}, B, T=0\right)$ phase diagram (Fig. 0.7 bottom). It should be noted that, at low fields $(B \lesssim 2 \mathrm{~T}), \sigma\left(n_{s}, B, T\right)$ curves exhibit beautiful scaling (Eng et al., 2002) in agreement with $T \rightarrow 0$ extrapolations and general considerations (Belitz and Kirkpatrick, 1994), providing additional strong evidence for a quantum phase transition in this system in parallel $B$. In analogy with 

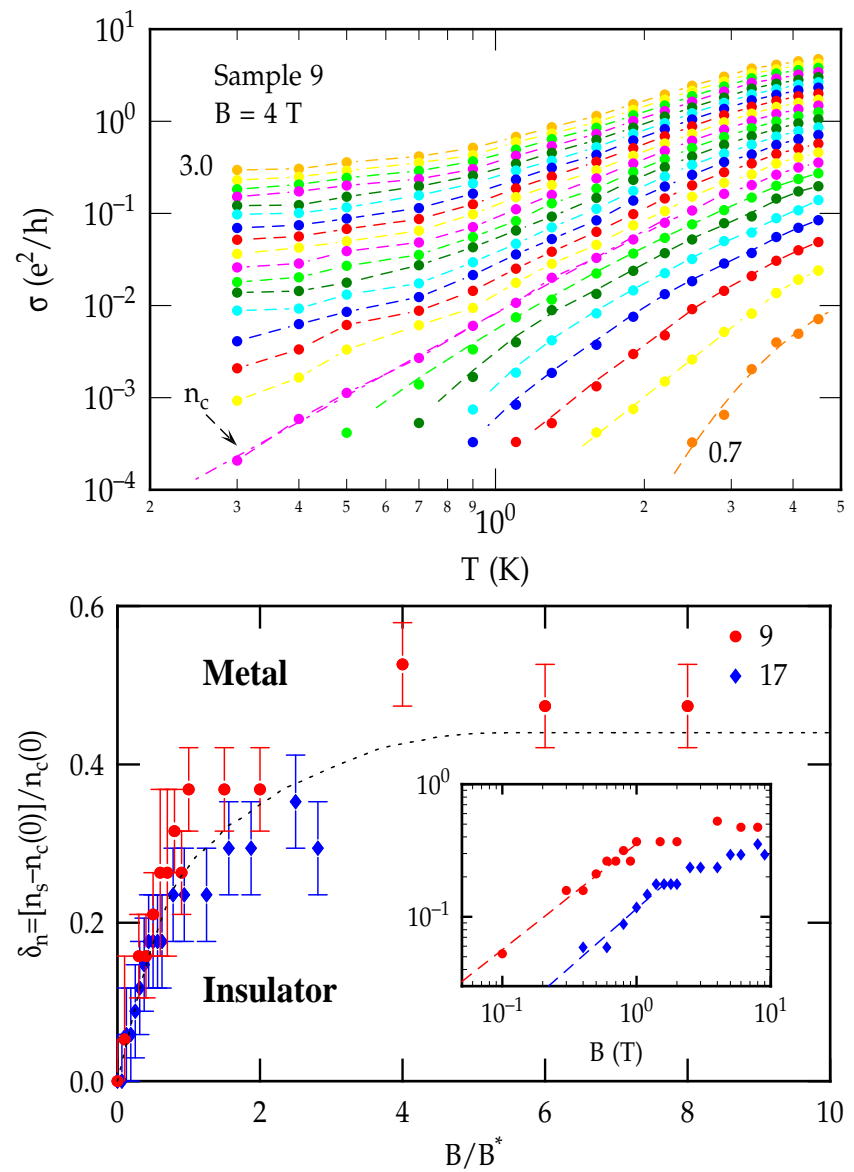

Fig. 0.7 Intermediate-mobility sample $\left(\mu \approx 1 \mathrm{~m}^{2} / \mathrm{Vs}\right)$ with local magnetic moments $\left(V_{\text {sub }}=+1 \mathrm{~V}\right)$ (Eng et al., 2002). Top panel: $\sigma(T)$ for sample 9 at $B=4 \mathrm{~T}$. $n_{s}$ varies from $3.0 \times 10^{11} \mathrm{~cm}^{-2}$ (top) to $0.7 \times 10^{11} \mathrm{~cm}^{-2}$ (bottom) in steps of $0.1 \times 10^{11} \mathrm{~cm}^{-2}$. $n_{c}(B=4 \mathrm{~T})=1.4 \times 10^{-11} \mathrm{~cm}^{-2}$ and is marked by the arrow. The dashed lines guide the eye. $\sigma_{c}$ clearly follows a simple power-law dependence on $T: \sigma_{c} \propto T^{x}$. Bottom panel: $T=0$ phase diagram for two samples. The dashed line guides the eye. The boundary between metallic and insulating phases is described by a power-law relation (see inset) $\left[n_{c}(B)-n_{c}(0)\right] / n_{c}(0) \propto\left(B / B^{*}\right)^{\beta}$ at low fields, with the same crossover exponent $\beta \approx 0.9$ for both samples $\left(B^{*}=1 \mathrm{~T}\right.$ for sample 9$)$. Inset: the same data vs. $B$ on a $\log -\log$ scale. The dashed lines are fits with the slopes equal to $\beta$. At $B=0, n_{c}\left(10^{11} \mathrm{~cm}^{-2}\right)=0.95 \pm 0.05$ $\left(r_{s} \approx 17\right)$ and $0.85 \pm 0.05\left(r_{s} \approx 18\right)$ for samples 9 and 17 , respectively.

high-mobility samples, the MR exhibits a strong increase in the region of the magneticfield driven MIT near $n_{c}(B)$ (Eng et al., 2001). For $B>2 \mathrm{~T}$, where 2DES is spin polarized, $n_{c}(B)$ saturates, indicating that the MIT occurs between a spin-polarized metal and a spin-polarized insulator. The existence of the metallic phase at fields up to 
$18 \mathrm{~T}$ has been confirmed by the measurements of $\sigma(T)$, which retains a simple powerlaw form, albeit with a different exponent (Eng et al., 2002). The charge dynamics has not been studied in these samples yet.

It is interesting that the $n_{c}(B)$ dependence in samples with local moments (Fig. 0.7 bottom) is quite similar to that obtained on high-mobility samples [Fig. 0.6(a)]. In both cases, at low fields $n_{c}(B)$ increases with $B$ in a power-law fashion: $\left[n_{c}(B)-\right.$ $\left.n_{c}(0)\right] / n_{c}(0) \propto B^{\beta}$. The crossover exponent $\beta \approx 0.9-1$ in Fig. 0.7 (bottom panel) and $\beta=1.1 \pm 0.1$ in Fig. 0.6(a). In other words, $n_{c}$ increases approximately linearly with $B$ at low fields. It is remarkable that this dependence is essentially the same in both cases, even though the $d \sigma / d T$ behaviors in the metallic phase at $B=0$ are strikingly different. The key features of the $n_{c}(B)$ phase diagram have been reproduced theoretically based on a scenario of quantum melting of a Wigner crystal as the mechanism of the MIT in sufficiently clean samples (Camjayi et al., 2008). In general, a power-law shift of $n_{c}$ with $B$ is expected to occur in the case of a true MIT (Belitz and Kirkpatrick, 1994), and has been observed in several 3D systems (Rosenbaum et al., 1989: Bogdanovich et al., 1997: Sarachik et al., 1998: Watanabe et al., 1999).

\subsection{Glassy freezing of electrons in two dimensions}

Understanding the dynamics of glasses and other systems out of equilibrium is one of the most challenging and rapidly evolving topics in condensed matter research (see Barrat et al. 2003). Since vastly different types of systems exhibit similar behavior, it is tempting to search for common organizing principles and unified theoretical approaches. However, despite some progress, there are still no well established theoretical frameworks for treating nonequilibrium behavior, which "remains largely uncharted territory" (Committee on CMMP 2010, 2007). For example, although glassy behavior may dominate the low-temperature properties of many complex materials near quantum phase transitions (Miranda and Dobrosavljević, 2005) (Sec.0.1), such as the MIT, quantum glasses are even less understood than their classical counterparts. Experimental studies of charge or Coulomb glasses (Davies et al., 1982, Grünewald et al., 1982 Pollak and Ortuño, 1982; Davies et al., 1984; (Pollak, 1984), which are of particular relevance to the MIT, have been relatively scarce (Monroe et al., 1987: Ben-Chorin et al., 1993; Ovadyahu and Pollak, 1997: Vaknin et al., 1998: Vaknin et al., 2000: Vaknin et al., 2002 Orlyanchik and Ovadyahu, 2004: Ovadyahu, 2006a: Ovadyahu, 2006b; |Martinez-Arizala et al., 1997; Martinez-Arizala et al., 1998, Bielejec and Wu, 2001: Hernandez et al., 2003; Grenet, 2003 ; Lee et al., 2005: Grenet et al., 2007: Kar et al., 2003; Thorsmølle and Armitage, 2010), and mostly limited to insulating systems far from the MIT. Recent observations (Bogdanovich and Popović, 2002b; Bogdanovich and Popović, 2002a; Jaroszyński et al., 2002b; Jaroszyński et al., 2002 a Popović et al., 2003: Jaroszyński et al., 2004b: Jaroszyński et al., 2004a, Jaroszyński and Popović, 2006 Jaroszyński and Popović, 2007b Jaroszyński and Popović, 2007a) of glassiness in a 2DES in Si MOSFETs near the MIT open up opportunities for exploring glassy phenomena in this important regime over a wide range of all the relevant parameters.

There are two basic ways to explore the dynamics of glassy systems. The first one is to measure the response of the system to some kind of a perturbation. In a spin glass, for example, this would typically involve a study of the relaxation of the mag- 
netization following some combination of rapid cooling and a change in the applied magnetic field (see Vincent (2007) for a pedagogical review). The second one is to measure the fluctuations of an observable with time (i.e. noise), which provides information on correlation 5 . In spin glasses, transport noise measurements were required in order to provide definitive information on the details of glassy ordering and dynamics (Weissman, 1993). Both approaches have been used to probe the dynamics of the 2DES in Si, focussing on the conductivity $\sigma$ as the variable most relevant to the MIT. Measurements were performed on both high- and low-mobility Si MOSFETs, which also differ substantially in their geometry, size, and many other fabrication details, spanning essentially the entire range of Si technology. Thus the emergence of glassy dynamics proves to be a universal phenomenon in Si inversion layers, at least in the absence of disorder-induced local magnetic moments. The effect of local moments on charge dynamics still remains to be investigated.

\subsubsection{Fluctuations of conductivity}

The experimental protocol for measuring the $\sigma(t)$ fluctuations is simple, in principle: the measurement of $\sigma$ at a given $V_{g}$ or electron density $n_{s}$ is set up at high temperatures, the sample is then cooled to the measurement $T$, and $\sigma$ is measured as a function of time. At the end of the measurement, the sample is warmed up to a temperature that is so high that a subsequent cool-down to the same measurement $T$ would result in a reproducible value of the time-averaged $\langle\sigma(t)\rangle$ within the experimental uncertainty. The carrier density $n_{s}$ is changed at a high temperature, and the protocol is repeated for different $n_{s}$ and $T$. In practice, the measurement set-up itself is often somewhat complicated (Bogdanovich and Popović, 2002b Jaroszyński et al., 2002b; Popović et al., 2003) because it is important to rule out various extraneous effects, such as the fluctuations of $T, V_{g}$, or contacts, as possible sources of the measured noise. However, there are now a number of standard methods to accomplish this (see, e.g., Scofield (1987), Verbruggen et al. (1989)).

In both low- and high-mobility samples, $\sigma$ exhibits strong fluctuations with time at low $n_{s}$ and $T$. Figure 0.8 left shows the fluctuations of $(\sigma-\langle\sigma\rangle) /\langle\sigma\rangle$ in a lowmobility sample for several $n_{s}$ at $T=0.13 \mathrm{~K}$. It is striking that, for the lowest $n_{s}$, the fluctuation amplitude is of the order of $100 \%$. In addition to rapid, highfrequency fluctuations, slow changes over periods of several hours are also evident. The probability density function (PDF) of the fluctuations illustrates how the sample explores the free energy landscape (FEL). For $n_{s}<n_{g}$, the PDF is always not only non-Gaussian but also has a very complex structure that changes with time (Fig. 0.8 right). The PDF broadens with the increasing sampling time $t$, as the system has more time to explore the FEL. However, it explores it so slowly that it remains nonergodic on experimental time scales. For $n_{s}>n_{g}$, on the other hand, the PDFs become perfectly Gaussian on much shorter time scales, suggesting that the system reaches equilibrium.

\footnotetext{
${ }^{5}$ In equilibrium systems, the connection between spontaneous fluctuations of a variable and the response of such a variable to a small perturbation in its conjugated field is given by the fluctuationdissipation relation. See Leuzzi and Nieuwenhuizen (2008) for the review and discussion of thermodynamics of out-of-equilibrium systems.

${ }^{6}\langle\ldots\rangle$ represents averaging over time intervals of, typically, several hours.
} 

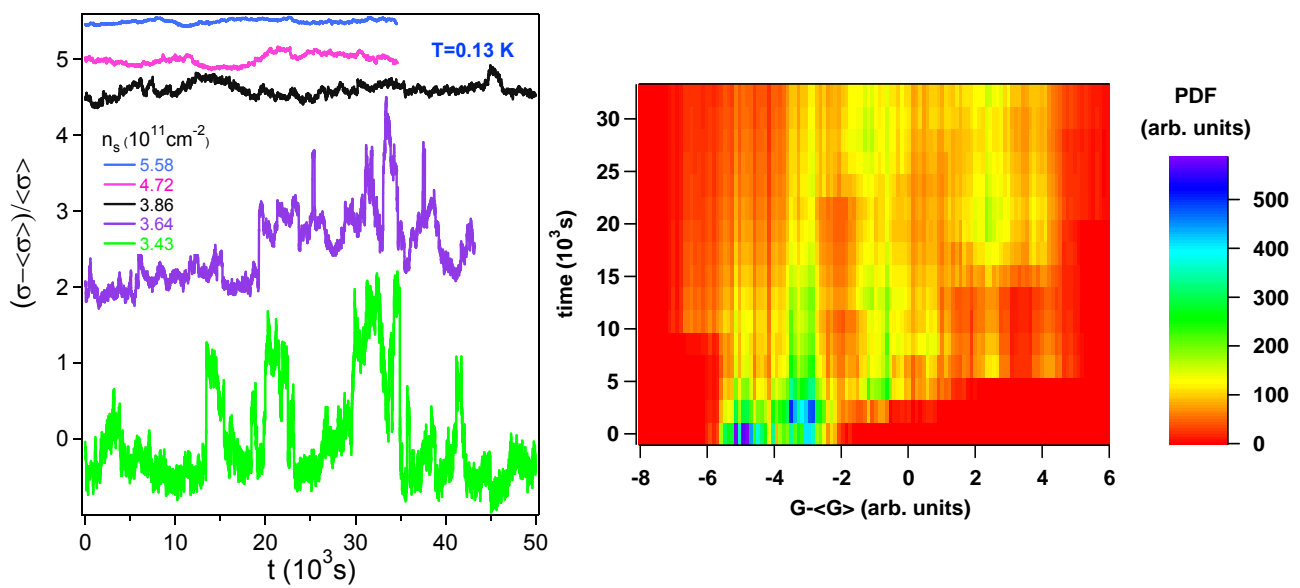

Fig. 0.8 Low-mobility sample (Bogdanovich and Popović 2002b and Fig. 0.5). Left: Relative fluctuations of $\sigma$ vs. time for different $n_{s}$ at $T=0.13 \mathrm{~K}$. Different traces have been shifted for clarity; the lowest $n_{s}$ is at the bottom and the highest $n_{s}$ at the top. Right: The color map of the probability density function (PDF) of the conductance $(G)$ fluctuations as a function of sampling time $t$ for the $n_{c}<n_{s}\left(10^{11} \mathrm{~cm}^{-2}\right)=5.58<n_{g}$ data shown on the left.

The amplitude of the fluctuations decreases dramatically from $~ 100 \%$ to less than $1 \%$ with increasing either $n_{s}$ (Fig. 0.8 left) or $T$.

An even more dramatic density dependence of the noise at low $T$ is revealed by the study of the power spectra $S(f)$ ( $f$-frequency) of the relative changes in the conductivity $(\sigma(t)-\langle\sigma\rangle) /\langle\sigma\rangle$. Most of the spectra were obtained in the $f=\left(10^{-4}-10^{-1}\right) \mathrm{Hz}$ bandwidth, where they follow the well-known empirical law $S \propto 1 / f^{\alpha}$ (Hooge, 1976. Weissman, 1988). At the highest $n_{s}, S(f)$ does not depend on $n_{s}$ (Fig. 0.9 left) but, as $n_{s}$ is reduced below $n_{g}, S$ increases enormously, by up to six orders of magnitude at low $f$. Moreover, for a given $n_{s}<n_{g}, S(f)$ increases exponentially with decreasing $T$ (Fig. 0.9 left, inset). The observed $d S / d T<0$ makes it possible to rule out various simple models of noise (see, e.g., Bogdanovich and Popović (2002b), Popović et al. (2003) for discussion). The most striking feature of the data, however, is the sharp jump of the exponent $\alpha$ at $n_{s} \approx n_{g}$ (Fig. 0.9 right). While $\alpha \approx 1$ ("pure" $1 / f$ noise) for $n_{s}>n_{g}, \alpha \approx 1.8$ below $n_{g}$, reflecting a sudden shift of the spectral weight towards lower frequencies. Similar large values of $\alpha$ have been observed in some spin glasses above the MIT (Jaroszyński et al., 1998; Neuttiens et al., 2000), and in submicron wires (Wróbel et al., 1998). Both the increase in the magnitude of the noise at low $f$ and the jump in $\alpha$ reflect a sudden and dramatic slowing down of the electron dynamics at $n_{g}$, indicating glassy freezing. The onset of glassy dynamics on the metallic side of the MIT, i.e. at $n_{g}>n_{c} \approx 5 \times 10^{11} \mathrm{~cm}^{-2}$, implies the existence of the metallic glass phase for $n_{c}<n_{s}<n_{g}$, which is consistent with the predictions of the model of interacting electrons near a disorder-driven MIT (Dobrosavljević et al., 2003). Since, in the glassy phase, $\alpha$ decreases with increasing $T$ (Fig. 0.9 right, inset), the large values of $\alpha$ and the jump in $\alpha\left(n_{s}\right)$ are observable only at relatively low $T$. 

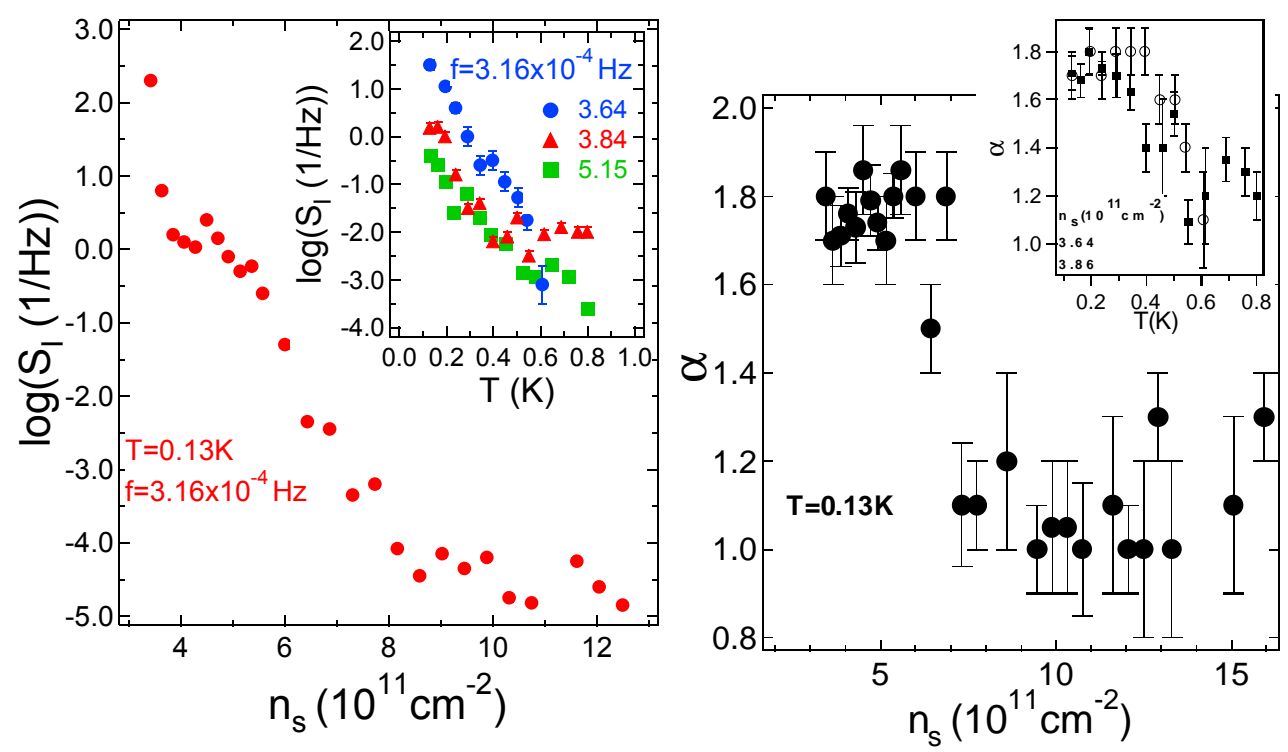

Fig. 0.9 Low-mobility sample Bogdanovich and Popović (2002b) and Fig. 0.5). Left: The normalized noise power $S\left(f=3.16 \times 10^{-4} \mathrm{~Hz}\right)$ vs. $n_{s}$ at $T=0.13 \mathrm{~K}$ (Bogdanovich and Popović, 2002a). Below $n_{g} \approx 7.5 \times 10^{11} \mathrm{~cm}^{-2}$, the noise increases exponentially with decreasing $n_{s}$. Inset: $S$ vs. $T$ for three different $n_{s}\left(10^{11} \mathrm{~cm}^{-2}\right)$ given on the plot. Right: At $n_{s} \approx n_{g}$, the exponent $\alpha$ exhibits a sharp jump from $\approx 1$ at high $n_{s}$ ("pure" $1 / f$ noise) to $\approx 1.8$ at low $n_{s}$ (Bogdanovich and Popović, 2002b). Inset: $\alpha$ vs. $T$ for two different $n_{s}\left(10^{11} \mathrm{~cm}^{-2}\right)(3.64$ - open symbols, 3.86 - solid symbols $)$ in the glassy phase (Popović et al., 2003).

Qualitatively the same behavior has been observed in the resistance (or conductance) noise of high-mobility samples, except that the glassy freezing takes place at $n_{g} \approx n_{c}$ (Jaroszyński et al., 2002b). Importantly, in both types of samples, the character of the noise also changes with density: while at low $n_{s}$ both the "shape" and the variance of the noise exhibit random, nonmonotonic changes with time, at high enough $n_{s}$ the noise always "looks" the same. This is illustrated in Fig. 0.10)(a) for a high-mobility sample. The figure shows $(\rho-\langle\rho\rangle) / \delta \rho$, where $\delta \rho=\left\langle(\rho-\langle\rho\rangle)^{2}\right\rangle^{1 / 2}$, in order to make it easier to compare the signals. A quantitative measure of the spectral wandering with time, such as that observed at low $n_{s}$, is the so-called second spectrum $S_{2}\left(f_{2}, f\right)$, which is a fourth-order noise statistic. $S_{2}$ is the power spectrum of the fluctuations of $S(f)$ with time (Weissman, 1993: Weissman et al., 1992), $i$. e. the Fourier transform of the autocorrelation function of the time series of $S(f)$. In general, if the fluctuators (e.g. two-level systems) are not correlated, $S_{2}\left(f_{2}, f\right)$ is white (independent of $f_{2}$ ) (Weissman et al., 1992, Weissman, 1988, Weissman, 1993) and equal to the square of the first spectrum. Such noise is called Gaussian. On the other hand, $S_{2}$ has a nonwhite character, $S_{2} \propto 1 / f_{2}^{1-\beta}$, for interacting fluctuators (Weissman et al., 1992 . 

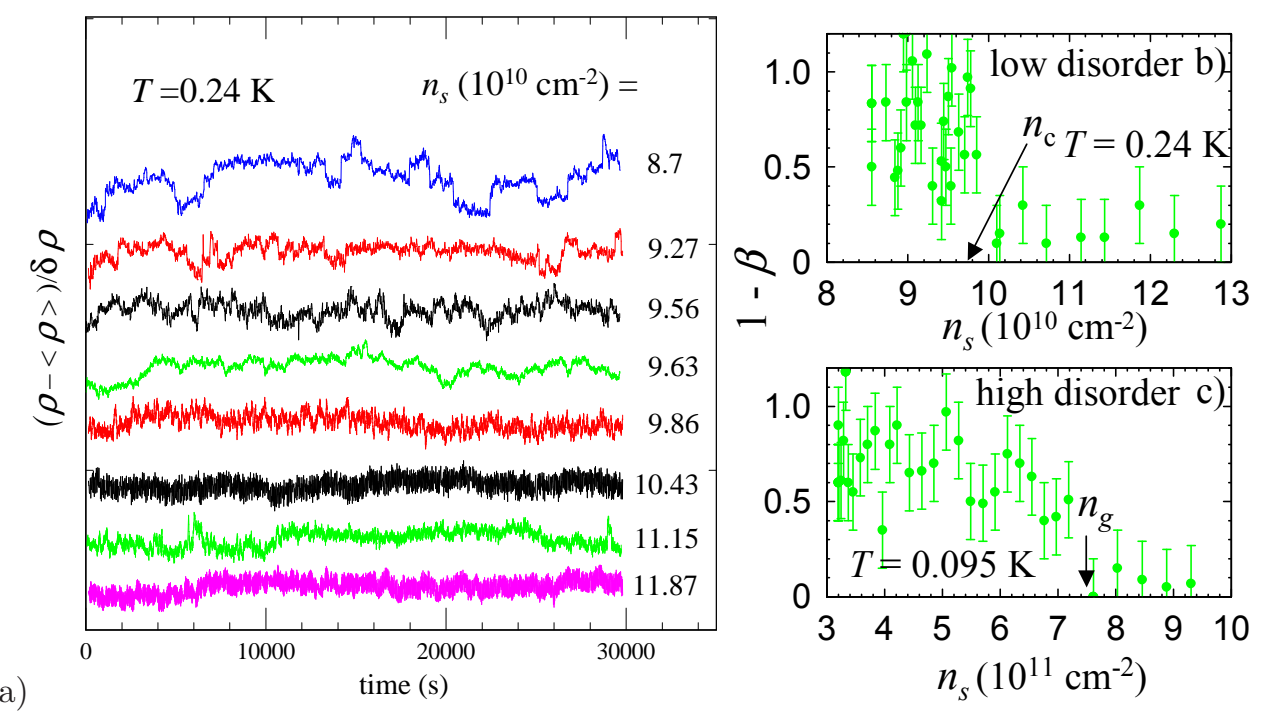

(a)

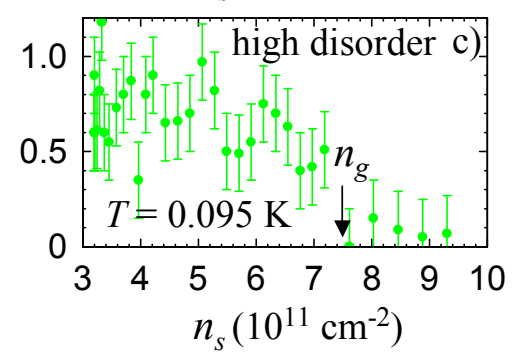

Fig. 0.10 (a) Resistance noise in a high-mobility (low-disordered) sample for several $n_{s}$ shown on the plot (adapted from Jaroszyński et al. 2002b). $(\rho-\langle\rho\rangle) / \delta \rho$ is plotted $\left(\delta \rho^{2}=\right.$ variance, $\rho$ - resistivity) in order to make the change in the character of the noise with $n_{s}$ more apparent. Different traces have been shifted vertically for clarity. Exponent $1-\beta$, a measure of correlations, $v$ s. $n_{s}$ for (b) high-mobility $\left[n_{c} \approx 9.7 \times 10^{10} \mathrm{~cm}^{-2}(\right.$ Jaroszyński et al., 2002b) $]$ and (c) low-mobility samples $\left[n_{c} \approx 5.0 \times 10^{11} \mathrm{~cm}^{-2}\right.$ (Bogdanovich and Popović, 2002b) $]$.

Weissman, 1988; Weissman, 1993). Therefore, the deviations from Gaussianity, or the exponent $(1-\beta)$, provide a direct probe of correlations between fluctuators. Indeed, $S_{2}$ has been an important tool in studies of other glasses.

A detailed dependence of the exponent $(1-\beta)$ on $n_{s}$ has been determined for both high- and low-mobility samples (Figs.0.10(b) and (c), respectively) (Jaroszyński et al., $2002 b)$.

In both cases, $S_{2}$ is white $[(1-\beta)=0]$ for $n_{s}>n_{g}$, indicating that the observed $1 / f$ noise results from uncorrelated fluctuators. It is quite remarkable that $S_{2}$ changes its character in a dramatic way exactly at $n_{g}$ in both types of samples. For $n_{s}<n_{g}, S_{2}$ is strongly non-Gaussian, which demonstrates that the fluctuators are strongly correlated and provides an unambiguous evidence for the onset of glassy dynamics in a 2DES at $n_{g}$.

In the studies of spin glasses, the scaling of $S_{2}$ with respect to $f$ and $f_{2}$ has been used (Weissman et al., 1992; Weissman, 1993) to unravel the glassy dynamics and, in particular, to distinguish generalized models of interacting droplets or clusters from hierarchical pictures. In the former case, the low- $f$ noise comes from a smaller number of large elements because they are slower, while the higher- $f$ noise comes from a larger number of smaller elements, which are faster. In this picture, which assumes compact droplets and short-range interactions between them, big elements are more likely to interact than small ones and, hence, non-Gaussian effects and $S_{2}$ will be 


\section{Contents}

stronger for lower $f . S_{2}\left(f_{2}, f\right)$, however, need to be compared for fixed $f_{2} / f$, $i$. e. on time scales determined by the time scales of the fluctuations being measured, since spectra taken over a fixed time interval average the high-frequency data more than the low-frequency data. Therefore, in the interacting "droplet" model, $S_{2}\left(f_{2}, f\right)$ should be a decreasing function of $f$ at constant $f_{2} / f$. In the hierarchical picture, on the other hand, $S_{2}\left(f_{2}, f\right)$ should be scale invariant: it should depend only on $f_{2} / f$, not on the scale $f$ (Weissman et al., 1992; Weissman, 1993). Figures 0.11(a) and (b) show that no systematic dependence of $S_{2}$ on $f$ is seen in either high- or low-mobility 2DES (Jaroszyński et al., 2002b). The observed scale invariance of $S_{2}\left(f_{2}, f\right)$ signals that the system wanders collectively between many metastable states related by a kinetic hierarchy. Metastable states correspond to the local minima or "valleys" in the FEL (Fig. 0.1), separated by barriers with a wide, hierarchical distribution of heights and, thus, relaxation times. Intervalley transitions, which are reconfigurations of a large number of electrons, thus lead to the observed strong, correlated, $1 / f$-type noise, remarkably similar to what was observed in spin glasses with a long-range correlation of spin configuration (Weissman et al., 1992, Weissman, 1993). On the other hand, in systems where both long-range and short-range interactions are present, such as lightly doped $\mathrm{La}_{2-x} \mathrm{Sr}_{x} \mathrm{CuO}_{4}, S_{2}\left(f_{2}, f\right)$ reveals clearly (Raičević et al., 2008, Raičević et al., 2011) [Fig. $0.11(\mathrm{c})]$ the presence of some characteristic energy scale, indicating that hierarchical models are not applicable and suggesting instead the formation of a cluster glass state. This is exactly what is expected in Coulomb systems with competing interactions (Sec. 0.1).

In the resistance noise measurements, the glass transition in a 2DES is thus manifested by (a) a sudden and dramatic increase of $S(f)$ and a jump of $\alpha$ from $\approx 1$ to $\approx 1.8$, indicating the slowing down of the dynamics, and (b) a change of the exponent $(1-\beta)$ from a white (zero) to a nonwhite (nonzero) value, indicating an abrupt change to a correlated statistics, consistent with the hierarchical pictures of glassy dynamics (Binder and Young, 1986). For high-mobility 2DES in Si MOSFETs, low- $T$ noise measurements were performed also in parallel $B$ (Jaroszyński et al., 2004b). By adopting the same criteria for the glass transition in $B$, it was possible to determine $n_{g}(B)$ shown in Fig. 0.6 (a) and to establish that charge, not spin, degrees of freedom are responsible for glassy ordering (see Sec. 0.2.2). Measurements in both $B=0$ and $B \neq 0$ show that glassy behavior generally emerges before the electrons localize (i.e. $n_{g}>n_{c}$ ), consistent with theory (Dobrosavljević et al., 2003). The glassy signatures in the noise become gradually stronger as $T$ decreases (e.g. Fig. 0.9 insets), suggesting that the glass transition takes place as $T \rightarrow 0$. Strong evidence for $T_{g}=0$ and further support for $n_{g}$ as the glass transition density is provided by the measurements of the response of the 2DES to a strong perturbation.

\subsubsection{Glassy response}

In MOSFET structures, the easiest and the most obvious way to excite the system is a sudden change of $V_{g}$. This method has been applied to several electron glasses (Ben-Chorin et al., 1993, Ovadyahu and Pollak, 1997, Vaknin et al., 1998; Vaknin et al., 2000, Vaknin et al., 2002; Orlyanchik and Ovadyahu, 2004: Ovadyahu, 2006a: Ovadyahu, 2006 b. Martinez-Arizala et al., 1997;:Martinez-Arizala et al., 1998;:Grenet, 2003: Grenet et al., 2007), 

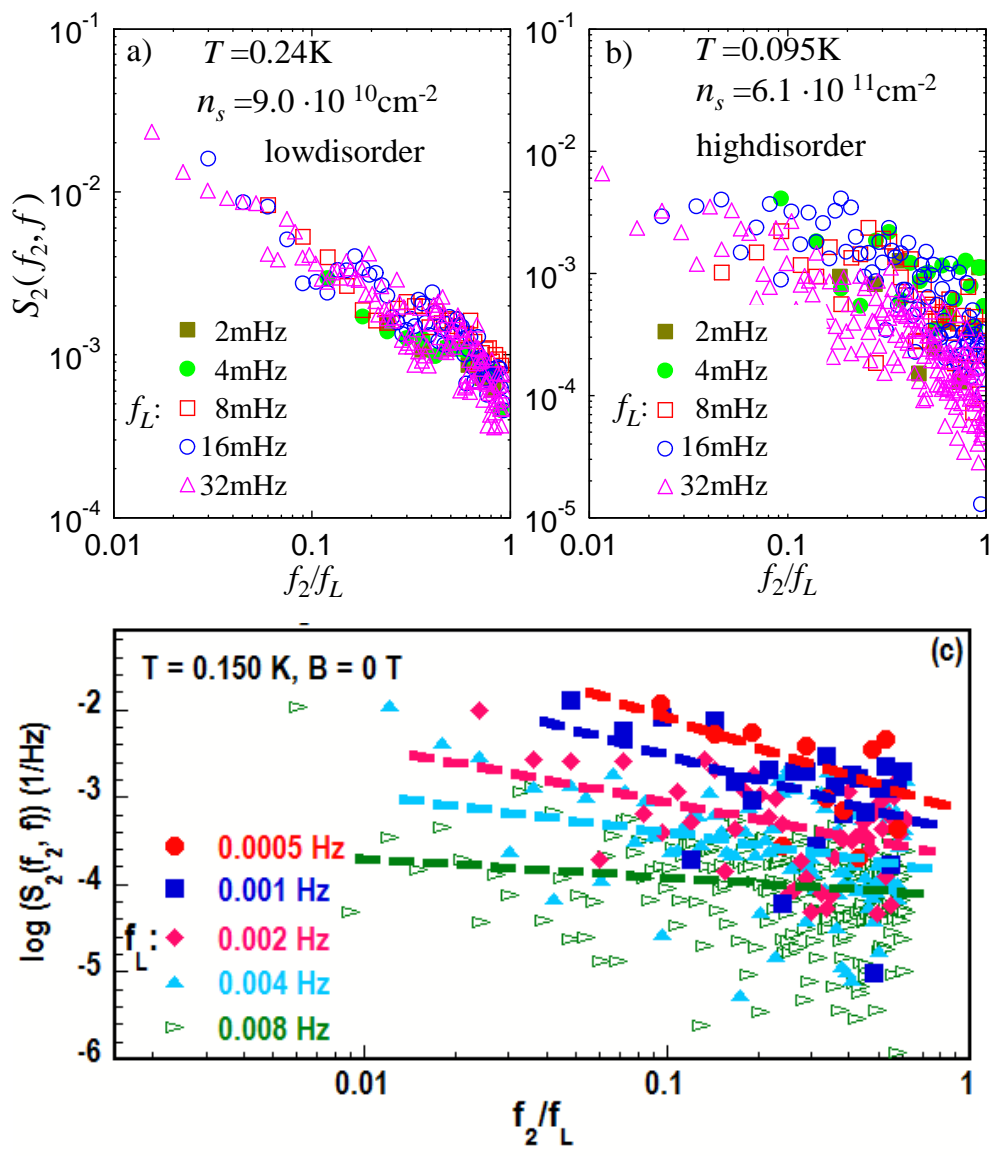

Fig. 0.11 Scaling of the second spectra $S_{2}$ measured at frequency octaves $f=\left(f_{L}, 2 f_{L}\right)$ for a given $n_{s}$ in (a) high-mobility (for $n_{s}<n_{c}$ ) and (b) low-mobility (for $n_{c}<n_{s}<n_{g}$ ) 2DES (Jaroszyński et al., 2002b). There is no characteristic time (or energy) scale observed, in agreement with hierarchical models. This is in contrast to (c) the insulating $\mathrm{La}_{1.97} \mathrm{Sr}_{0.03} \mathrm{CuO}_{4}$, where $S_{2}$ clearly shows the presence of some characteristic energy scale in that system (Raičević et al., 2008 Raičević et al., 2011), suggesting that charge carriers form a cluster glass state. Dashed lines are linear fits to guide the eye.

including a 2DES in Si (Jaroszyński and Popović, 2006)|Jaroszyński and Popović, 2007 b. Jaroszyński and Popović, 2007 a Jaroszyński and Popović, 2009). So far, only low-mobility samples discussed above (Secs. 0.2.1 and 0.3.1) have been probed in this way. In particular, three different experiments have been carried out, as described below.

Relaxations of conductivity after a rapid change of carrier density. A systematic study of the relaxations (i.e. time dependence) of the conductivity $\sigma(t)$ has been performed at different $n_{s}$ and $T$ after a large, rapid change of $n_{s}$ (controlled by $V_{g}$ ) (Jaroszyński and Popović, 2006). The sample was first cooled from high temperature 

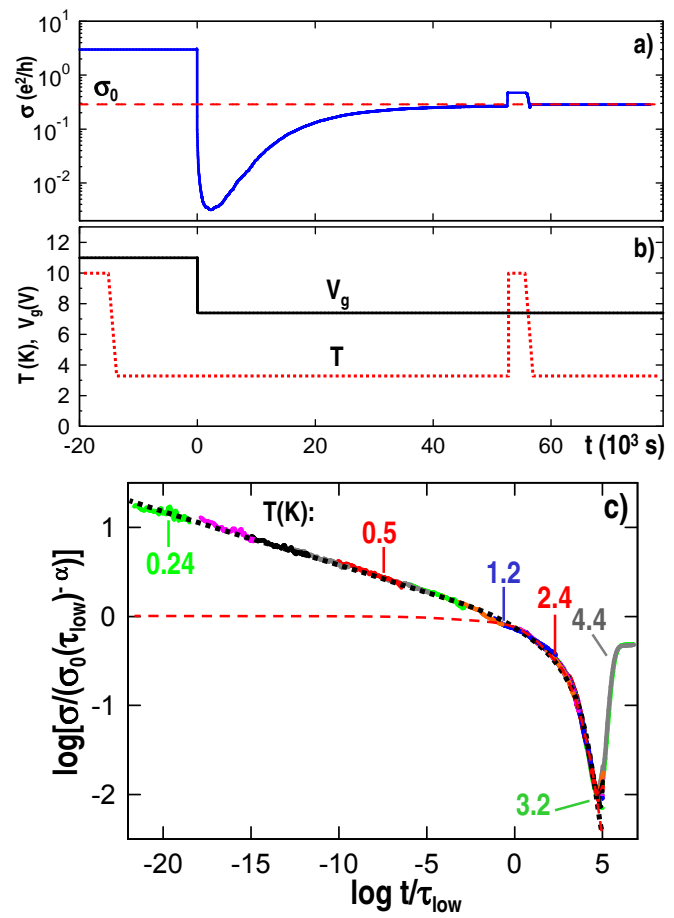

Fig. 0.12 Low-mobility sample with $n_{g} \approx 7.5 \times 10^{11} \mathrm{~cm}^{-2}$ and $n_{c} \approx 4.5 \times 10^{11} \mathrm{~cm}^{-2}$. (a) $\sigma(t)$ for $V_{g}^{i}=11 \mathrm{~V}\left[n_{s}\left(10^{11} \mathrm{~cm}^{-2}\right)=20.26\right], V_{g}^{f}=7.4 \mathrm{~V}\left[n_{s}\left(10^{11} \mathrm{~cm}^{-2}\right)=4.74\right]$, and $T=3.3 \mathrm{~K}$. (b) Experimental protocol: $V_{g}(t)$ and $T(t)$. (c) All the data at short times, (i.e. before the minimum) are consistent with the Ogielski relaxation, $\sigma(t, T) / \sigma_{0}(T) \propto\left(\tau_{\text {low }}\right)^{-\alpha}\left(t / \tau_{\text {low }}\right)^{-\alpha} \exp \left[-\left(t / \tau_{\text {low }}\right)^{\beta}\right]$ (dotted line). The lowest $T$ data clearly deviate from the stretched exponential dependence (dashed line). The data have been collapsed with respect to the $2.4 \mathrm{~K}$ curve. Adapted from Jaroszyński and Popović 2006.

$(10 \mathrm{~K})$ to the measurement temperature $T$ with an initial gate voltage $V_{g}^{i}$. Then, at $t=0$, the gate voltage was switched rapidly (within $1 \mathrm{~s}$ ) to a final value $V_{g}^{f}$, and $\sigma\left(t, V_{g}^{f}, T\right)$ was measured. In general, the results did not depend on any of the following: initial temperature, as long as it was $\geq 10 \mathrm{~K} ; V_{g}^{i}$; the cooling time, which was varied between 30 minutes and 10 hours; the time the sample was kept at $10 \mathrm{~K}$ (from 5 minutes to 8 hours); the time the sample spent at the measurement $T$ before $V_{g}$ was changed (from 5 minutes to 8 hours). Figures 0.12 (a) and (b) show a typical experimental run with $\sigma(t>0)$ exhibiting a rapid $(<10 \mathrm{~s})$ initial drop followed by a slower relaxation 7 . After going through a minimum, $\sigma(t)$ increases and approaches a value $\sigma_{0}\left(V_{g}^{f}, T\right)$. A subsequent warm-up to $10 \mathrm{~K}$ and a cool down to the same measurement $T=3.3 \mathrm{~K}$, while keeping the gate voltage fixed at $V_{g}^{f}$, shows that $\sigma_{0}\left(V_{g}^{f}, T\right)$ represents the equilibrium conductivity corresponding to the given $V_{g}^{f}$ and

\footnotetext{
${ }^{7}$ The RC charging times of the device and the circuit were at most $10 \mathrm{~ms}$.
} 
$T$. It is interesting that, even though initially it drops to a value close to $\sigma_{0}, \sigma$ first goes away from equilibrium before it starts approaching $\sigma_{0}$ again. At the end of the run, the sample was warmed up to $10 \mathrm{~K}$, gate voltage changed back to the same $V_{g}^{i}$, and the experiment was repeated at a different $T$ for the same $V_{g}^{f}$. The whole process was then repeated for different values of $V_{g}^{f}$, in order to map out the density dependence of various relaxation parameters. It was established (Jaroszyński and Popović, 2006) that, for $n_{s}<n_{g}$, the relaxations have the following properties.

After a sufficiently long $t, \sigma$ relaxes exponentially to its equilibrium value $\sigma_{0}$. The corresponding equilibration time obeys the simply activated form $\tau_{e q} \propto \exp \left(E_{a c t} / T\right)$, $E_{a c t} \approx 57 \mathrm{~K}$. While the microscopic origin of the activation energy $E_{a c t}$ is not known yet, the activation to an upper subband in $\mathrm{Si}$ MOSFETs or to $\mathrm{Si}_{-} \mathrm{SiO}_{2}$ interface traps has been ruled out (Jaroszyński and Popović, 2007b). However, regardless of the equilibration mechanism, the important result is that $\tau_{e q} \rightarrow \infty$ as $T \rightarrow 0$, so that, strictly speaking, the system cannot reach equilibrium only at $T=0$. In other words, the glass transition takes place at $T_{g}=0$. Remarkably, a Monte Carlo study of the 2D Coulomb glass model has also found (Grempel, 2004) an exponential divergence of $\tau_{e q}$, signaling a glass transition at $T_{g}=0$. There are, however, some differences in the detail between the model and the experiment, indicating a need for further refinement of theory.

For short enough $t, \sigma(t)$ obeys a nonexponential, Ogielski form (Ogielski, 1985) $\sigma(t, T) / \sigma_{0}(T) \propto t^{-\alpha} \exp \left[-\left(t / \tau_{\text {low }}\right)^{\beta}\right]\left(0<\alpha\left(n_{s}\right)<0.4,0.2<\beta\left(n_{s}\right)<0.45\right)$ [Fig. [0.12(c)], which is a product of a power law and a stretched exponential function. Both types of relaxations are considered to be typical signatures of glassy behavior and reflect the existence of a broad distribution of relaxation times. The scaling parameter $\tau_{\text {low }} \propto$ $\exp \left(E_{a} / k T\right)\left(E_{a} \approx 20 \mathrm{~K}\right)$ so that, as $T \rightarrow 0, \tau_{\text {low }} \rightarrow \infty$ and the relaxations attain a pure power-law form $\propto t^{-\alpha}$. The Ogielski form, the divergence of $\tau_{\text {low }}$, and the resulting power law relaxation at $T_{g}$ are consistent with the general scaling arguments (Hohenberg and Halperin, 1977) near a continuous phase transition occurring at $T_{g}=0$. These results are very similar to scaling observed in spin glasse: 8 above $T_{g}$ (Pappas et al., 2003). In a 2DES, $\alpha \rightarrow 0$ as $n_{s} \rightarrow n_{g}$, providing further evidence for $n_{g}$ as the glass transition density.

$\tau_{\text {low }}$ exhibits a very pronounced and precise dependence on the density: $\tau_{\text {low }} \propto$ $\exp \left(\gamma n_{s}^{1 / 2}\right)\left(\gamma-\right.$ a proportionality constant). Since $1 / r_{s}=E_{F} / U \propto n_{s}^{1 / 2}$ in $2 \mathrm{D}$ (Sec. [0.2), this particular form of $\tau_{\text {low }}\left(n_{s}\right)$ provides strong evidence that the observed out-ofequilibrium behavior is dominated by the Coulomb interactions between $2 \mathrm{D}$ electrons.

Perhaps the most peculiar finding is that the 2DES equilibrates only after it first goes farther away from equilibrium. While the precise mechanism for nonmonotonic relaxation remains controversial, studies of other materials [spin glasses (Jonsson et al., 1999 . Jönsson and Takayama, 2005), manganites (Levy et al., 2002), insulating granular metals (Kurzweil and Frydman, 2007), biological systems (Nelson, 2003)] and some theoretical models (Morita and Kaneko, 2005: Miyashita et al., 2007) suggest that it may be a general feature of systems that are far from equilibrium.

It should be noted that it is quite remarkable that this one experiment has provided so many important results. To put things into a perspective, for example, it has not

\footnotetext{
${ }^{8} T_{g}$ is finite in spin glasses.
} 


\section{Contents}

been possible to determine $T_{g}$ in other electron glasses so far, and scaling in spin glasses (Pappas et al., 2003) has been observed only relatively recently, in spite of many more years of study.

Relaxations of conductivity after a waiting time protocol: aging and memory loss. A key characteristic of relaxing glassy systems is the loss of time translation invariance, reflected in aging effects (Struik, 1978, Hodge, 1995, Rubi and Perez-Vicente, 1997). The system is said to exhibit aging if its response to an external excitation depends on the system history in addition to the time $t$. In a systematic study of the history dependence in a 2DES (Jaroszyński and Popović, 2007b), $\sigma(t)$ was measured after the temporary change of $n_{s}$ during the waiting time $t_{w}$ [Figs. 0.13(a) and (b)]. The history was varied by changing $t_{w}$ and $T$ for several initial (final) $n_{s}$.

Two types of response have been observed: 1) monotonic, for relatively "small" excitations, where $\sigma(t)$ depend on $t_{w}$, i.e. 2DES shows aging; 2) nonmonotonic, for sufficiently "large" excitations, where $\sigma(t)$ "overshoots" $\sigma_{0}$ (i.e. it first goes farther away from equilibrium) and relaxations no longer depend on $t_{w}$ (memory loss). The monotonic relaxations [Fig. 0.13(a)] are consistent with a power-law form at the shortest times (or lowest $T$ ) and, at the longest $t$, with a simple exponential approach to equilibrium.

The aging is observed when $t_{w} \ll \tau_{e q}(T)$, i.e. if the system is unable to equilibrate under the new conditions during $t_{w}[$ Fig. 0.13(c)]. In that case, $\sigma(t)$ depends also on $t_{w}$ : the system has a memory of the time $t_{w}$. This is very similar to spin glasses, where $T$ or $B$ play a role analogous to that of $n_{s}$. In the opposite case, when $\tau_{e q}(T) \ll t_{w}$ and the 2DES equilibrates at a new state, the relaxations do not depend on $t_{w}$ since the system, naturally, has no memory of the waiting time. Therefore, the overshooting is observed when the system is excited out of thermal equilibrium. This is analogous to the experiment described above (Sec. 0.3.2) and thus sheds some light on that intriguing phenomenon.

The gate voltage changes $\Delta V_{g}$ employed in the relaxation experiments in a $2 \mathrm{DES}$ have been relatively large. For example, $n_{s}$ was changed up to a factor of 7 , and thus the 2DES could go from the conducting to the insulating regime. Such large $\Delta V_{g}$ are expected to trigger major rearrangements of the electron configuration since the corresponding shifts of the Fermi energy $\Delta E_{F} \gg k_{B} T$ (Müller and Lebanon, 2005). It is possible to speculate that such large perturbations might be somehow responsible for the peculiar overshooting effect. Considerable charge rearrangements, coupled with possibly substantial changes in the screening of the 2DES across the MIT (Pastor and Dobrosavljević, 1999; Müller and Ioffe, 2004:Pankov and Dobrosavljević, 2005), present a fundamentally different situation from the cooling of the 2DES at a fixed $n_{s}$ when $k_{B} \Delta T \ll E_{F}$, where no relaxations have been observed. On the other hand, smaller perturbations of a Coulomb glass are expected to lead to memory effects (Lebanon and Müller, 2005), in agreement with the observations for $t_{w} \ll$ $\tau_{e q}(T)$. In that case, the final state has a large configurational similarity with the original state due to the shortness of $t_{w}$. In $\mathrm{InO}_{x}$ films, another well-studied electron glass, the overshooting of equilibrium has not been seen, but aging and mem-

$$
{ }^{9} E_{F}[\mathrm{~K}]=7.31 n_{s}\left[10^{11} \mathrm{~cm}^{-2}\right] \text { for electrons in Si (Ando et al., 1982). }
$$



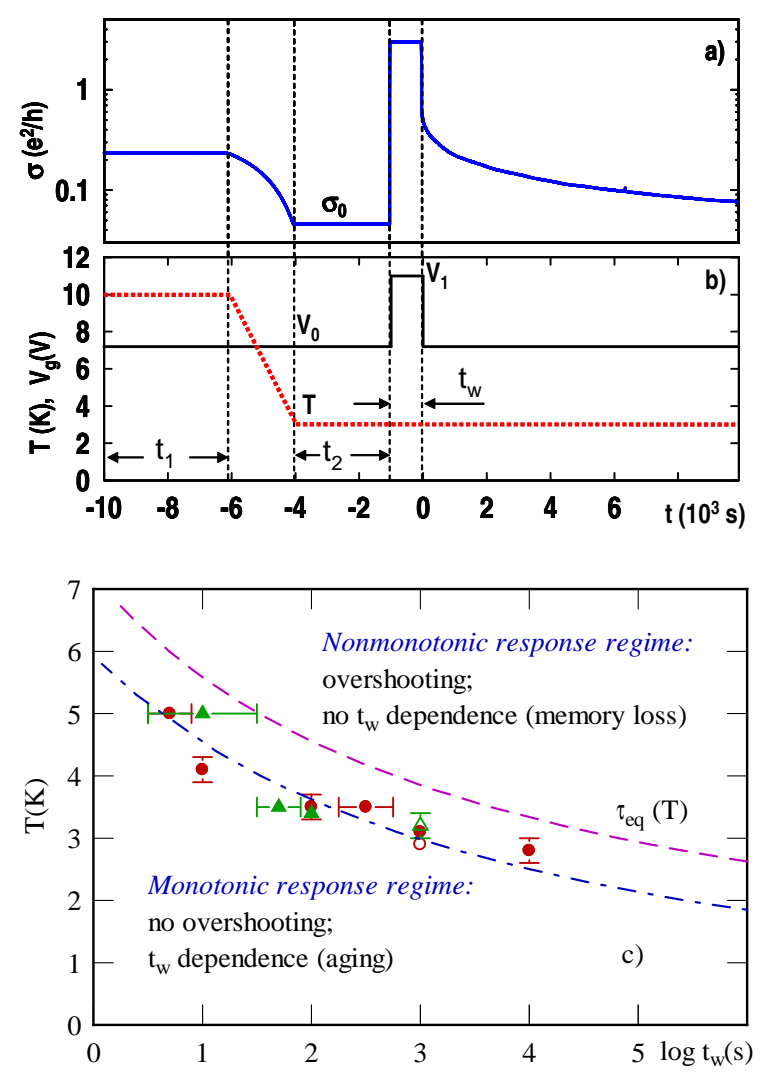

Fig. $0.13(\mathrm{a}) \sigma(t)$ for $V_{0}=7.2 \mathrm{~V}\left[n_{s}\left(10^{11} \mathrm{~cm}^{-2}\right)=3.88<n_{c}\right], V_{1}=11 \mathrm{~V}$ $\left[n_{s}\left(10^{11} \mathrm{~cm}^{-2}\right)=20.26\right], t_{w}=1000 \mathrm{~s}$, and $T=3.0 \mathrm{~K}$. (b) The corresponding experimental protocol: $V_{g}(t)$ and $T(t)$. The results do not depend on the cooling time (varied from 30 minutes to 10 hours), nor on $t_{1}$ and $t_{2}$ (varied from 5 minutes to 8 hours each). (c) The symbols show the values of $\left(T, t_{w}\right)$ where the overshooting vanishes, i.e. the conditions that separate the two regimes. Open and solid symbols correspond to different samples; the blue dot-dashed line guides the eye. The purple dashed line represents $\tau_{e q}(T)$. Adapted from Jaroszyński and Popović (2007b).

ory effects have been observed (Ben-Chorin et al., 1993, Ovadyahu and Pollak, 1997; Vaknin et al., 1998; Vaknin et al., 2000; Vaknin et al., 2002). Those experiments were done in the regime of small perturbations, because the typical change in the carrier density due to $\Delta V_{g}$ was $\sim 1 \%$, the system always remained deep in the insulating state, and $t_{w} \ll \tau_{e q}$ was satisfied.

Aging effects across the MIT in 2D. Aging effects have been instrumental as a probe of complex nonequilibrium dynamics in many types of materials. In a 2DES, where the onset of glassy dynamics takes place on the metallic side, a study of aging, especially across the MIT, is of great interest. Aging is observed if $t_{w} \ll \tau_{e q}$ [Fig. [0.13 (c)]. 
Since the equilibration time $\tau_{e q}$ diverges exponentially as $T \rightarrow 0$ (Sec. 0.3.2), strictly speaking, the system can reach equilibrium at all $T>0$. However, even at $T$ that are not too low $(e . g . \sim 1 \mathrm{~K}), \tau_{e q}$ exceeds easily not only the experimental time window but also the age of the Universe (Jaroszyński and Popović, 2006). This makes it relatively easy to study the out-of-equilibrium relaxation of $\sigma$ at times $t \ll \tau_{e q}$, where one expects to find properties common to other types of glasses. In strongly localized systems, such as $\mathrm{InO}_{x}$ films, the aging function $\sigma\left(t, t_{w}\right)$ is just a function of $t / t_{w}$ (Ben-Chorin et al., 1993; Ovadyahu and Pollak, 1997, Vaknin et al., 1998, Vaknin et al., 2000, Vaknin et al., 2002). This is known as simple, or full aging. It is interesting that, in spin glasses, full aging has been demonstrated only relatively recently (Rodriguez et al., 2003). In general, however, the existence of a characteristic time scale $t_{w}$ does not necessarily imply simple $t / t_{w}$ scaling (Barrat et al., 2003). In the mean-field models of glasses, for example, two different cases are distinguished: one, where full aging is expected, and the other, where no $t / t_{w}$ scaling is expected (Bouchaud et al., 1997). Experimentally, departures from full aging are common (Struik, 1978. Rubi and Perez-Vicente, 1997).

Aging was investigated in detail in a 2DES (Jaroszyński and Popović, 2007a) using the waiting time protocol [Sec. 0.3.2 Figs. 0.14(a), (b)], but $T$ was kept fixed at $1 \mathrm{~K}$ such that $\tau_{e q}$ was astronomical and, hence, the 2DES was always deep in the $t_{w} \ll \tau_{e q}$ limit [Fig. 0.13(c)]. $\sigma\left(t, t_{w}\right)$ were then explored systematically both as a function of final $n_{s}$ and of the difference in densities during and after $t_{w}$. Figure 0.14(c) illustrates the significant effect of $t_{w}$ on $\sigma(t)$. In fact, all the $\sigma\left(t, t_{w}\right)$ data can be collapsed onto a single curve simply by rescaling the time axis by $t_{w}$ [Fig. 0.14(d)]. Therefore, in this case, the system exhibits full aging at least up to $t \approx(2-3) t_{w}$. The relaxations can be described by a power law $\sigma(t) / \sigma_{0} \propto\left(t / t_{w}\right)^{-\alpha}$ for times up to about $t_{w}$, followed by a slower relaxation at longer $t$. This means that the memory of $t_{w}$ is imprinted on the form of each $\sigma(t)$.

For all $n_{s}<n_{c}, \sigma\left(t, t_{w}\right)$ exhibit simple or full aging. However, as $n_{s}$ increases above $n_{c}$, there is an increasingly strong departure from full aging. In some other glasses (Struik, 1978; Alba et al., 1986, Alba et al., 1987), it was found that the data could be scaled with a modified waiting time $\left(t_{w}\right)^{\mu}$, where $\mu$ is a fitting parameter ( $\mu=1$ for full aging). Even though $\mu$ may not have a clear physical meaning, the $\mu$-scaling approach has proved to be a useful tool for studying departures from full aging (Struik, 1978, Barrat et al., 2003). By adopting a similar method in the study of aging in a 2DES, it was possible to achieve an approximate collapse of the data (Jaroszyński and Popović, 2007a). The plot of $\mu$ vs. $n_{0}$ [Fig. 0.14(e)] shows a clear distinction between the full aging regime for $n_{s}<n_{c}$, and the aging regime where significant departures from full scaling are seen. It is striking that the largest departure occurs at $n_{s} \approx n_{g}$. For $n_{s}>n_{g}$, some small relaxations are observed (only for $k_{F} l<1$ ) resulting from finite-temperature effects, but they vanish in the $T \rightarrow 0$ limit (Sec. 0.3.2). It was also determined that $\mu$ does not depend on $T$ (Jaroszyński and Popović, 2009).

These results are striking because they reveal an abrupt change in the nature of the glassy phase exactly at the 2D MIT itself, before glassiness disappears completely at a higher density $n_{g}$. In other words, this is strong evidence that the insulating glassy phase and the metallic glassy phase are different. Therefore, the difference in the aging 

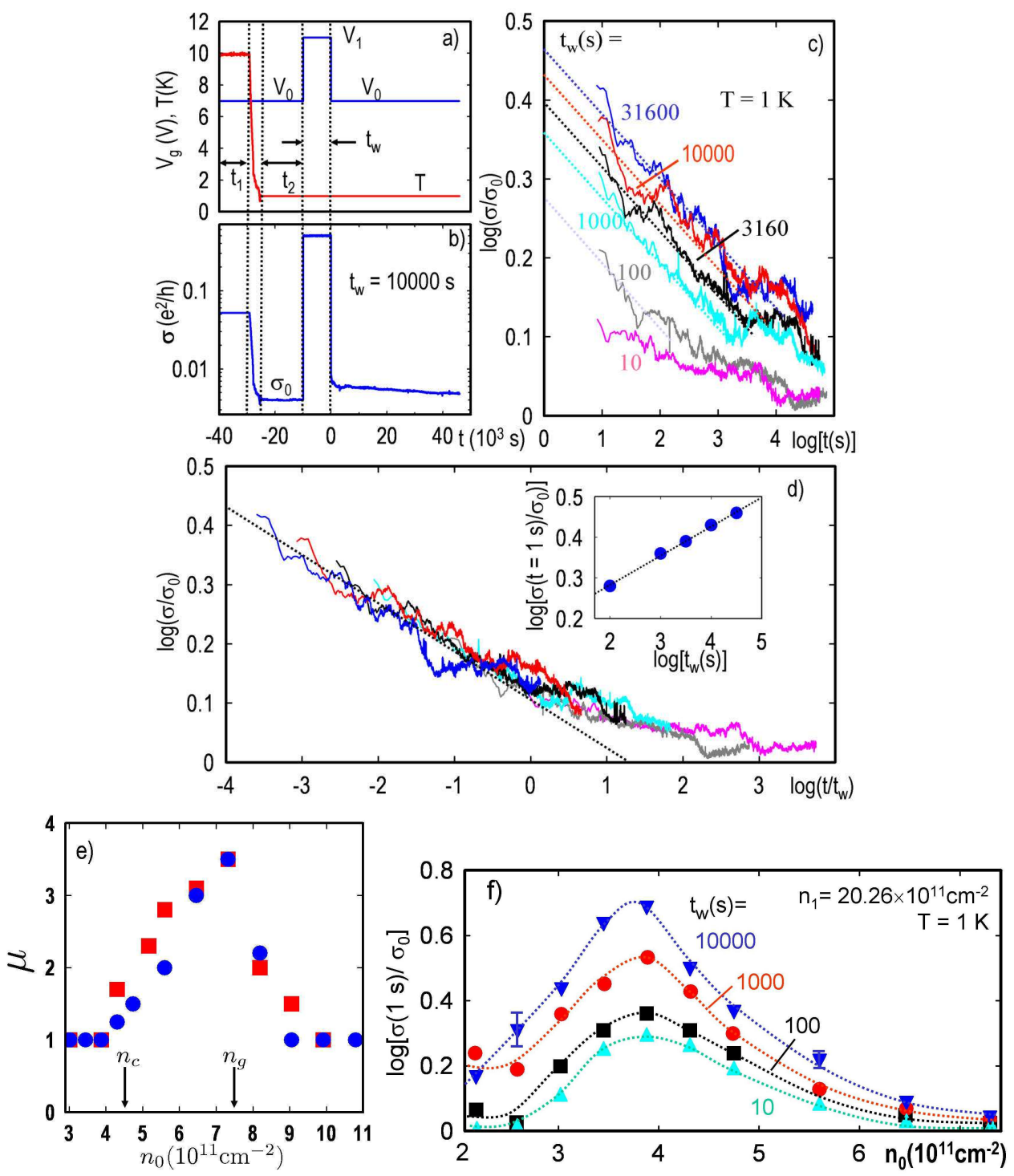

Fig. 0.14 (a) $V_{g}(t)$ and $T(t)$ in a typical experimental protocol, which always starts with the 2DES in equilibrium at $10 \mathrm{~K}$ (Jaroszyński and Popović, 2007b). (b) The corresponding $\sigma(t)$. The relaxation of $\sigma$ during $t_{w}$ is too small to be seen on this scale. (c) $\sigma(t>0)$ for several $t_{w}$, as shown; $V_{0}=7.0 \mathrm{~V}\left[n_{0}\left(10^{11} \mathrm{~cm}^{-2}\right)=3.02<n_{c}\right], V_{1}=11 \mathrm{~V}\left[n_{1}\left(10^{11} \mathrm{~cm}^{-2}\right)=20.26\right]$. The dotted lines are linear fits for $t \leq t_{w}$. (d) The same data as in (c) but plotted $v s . t / t_{w}$. The dotted line is a fit for $t \leq t_{w}$ with the slope $-\alpha=-0.081 \pm 0.005$. Inset: $\sigma(t=1 \mathrm{~s}) / \sigma_{0} v s . t_{w}$. The dotted line is a fit with the slope $\alpha=0.076 \pm 0.005$. (e) $\mu$ vs. $n_{0}$ for two samples. $\mu$ does not depend on $n_{1}$. (f) Relaxation amplitudes vs. $n_{0}$ for several $t_{w}$. Dotted lines guide the eye. Adapted from Jaroszyński and Popović (2007a), and Jaroszyński and Popović (2009). 
properties below and above $n_{c}$ puts constraints on the theories of glassy freezing and its role in the physics of the $2 \mathrm{D}$ MIT.

Furthermore, for a given $t_{w}$, the amplitude of $\sigma(t) / \sigma_{0}$ has a peak at $n_{s} \lesssim n_{c}$ [Fig. 0.14(f)], reflecting an interesting and surprising suppression of the relaxations on the insulating side of the 2D MIT. While a clear understanding of this effect is lacking, it is plausible that collective charge rearrangements that are responsible for the slow dynamics will be suppressed as the 2DES becomes strongly localized. It would be also of interest to study aging deeper in the insulator, in the variable-range hopping regime, but that is not possible because of the small relaxation amplitudes and the large intrinsic sample noise. Of course, the effects of disorder could be explored further by extending the relaxation studies to cleaner (high-mobility) 2DES, where $n_{g} \gtrsim n_{c}$ (Jaroszyński et al., 2002b, Jaroszyński et al., 2004b). Regardless of the origin of the nonmonotonic behavior in Fig. 0.14(f), however, it is important to note that it reflects another change in the aging properties that occurs at the MIT.

\subsection{Summary}

Careful studies have provided strong evidence that all 2DES in Si MOSFETs exhibit a metal-insulator transition regardless of the amount or type of disorder (Fig. 0.15). This is confirmed by extrapolating $\sigma(T)$ on both metallic and insulating sides of the MIT, and by dynamical scaling of $\sigma\left(n_{s}, T\right)$ over a wide range of parameters in both $B=0$ and $B \neq 0$. In $B=0$, the scaling form $\sigma\left(n_{s}, T\right)=\sigma_{c}(T) f\left(T / \delta_{n}^{z \nu}\right)$ is obeyed with a temperature dependent critical conductivity $\sigma_{c}=\sigma\left(n_{s}=n_{c}, T\right) \propto T^{x}$, where the value of $x$ depends on the disorder. Thus $\sigma_{c}(T)$ belongs to the insulating family of curves. The results are consistent with general arguments near the MIT (Belitz and Kirkpatrick, 1994) and they are similar to the behavior in doped semiconductors near the 3D MIT (Sarachik, 1995). The 2D metallic phase survives in very high parallel magnetic fields, long after the 2DES becomes fully spin polarized.

Measurements of the charge dynamics in a 2DES have established that the glass transition takes place at $T_{g}=0$ for all $n_{s}<n_{g}$ (Fig. 0.15). In general, the glassiness sets in on the metallic side of the MIT, i.e. at a density $n_{g}>n_{c}$, thus giving rise to an intermediate, metallic glass phase between the "normal" metal (i.e. in the $k_{F} l>1$ regime) and the glassy insulator. The glass transition and various glassy effects are observable only for $k_{F} l<1$, so that the intermediate phase is a very poor metal $\left(\sigma(T=0) \neq 0 \ll e^{2} / h\right)$. It is characterized by a very specific, non-Fermi liquid $T^{3 / 2}$ correction to $\sigma$ in both low-mobility samples in $B=0$ and high-mobility samples in a magnetic field. In high-mobility samples in $B=0$, the intermediate phase practically vanishes and the glass transition coincides with the MIT.

The manifestations of the glass transition in a 2DES for $n_{s}<n_{g}$, as demonstrated by resistance noise measurements, include a dramatic slowing down of the electron dynamics and correlated statistics consistent with the hierarchical picture of glasses. The results have been further confirmed and supported by the studies of relaxations, which provide evidence for the diverging equilibration time as $T \rightarrow 0$, broad distribution of relaxation times in the system, and scaling (in the time domain), consistent with a continuous phase transition at $T_{g}=0$. The aging, one of the hallmarks of glassy dynamics, shows an abrupt change in its properties at the MIT, indicating that the 


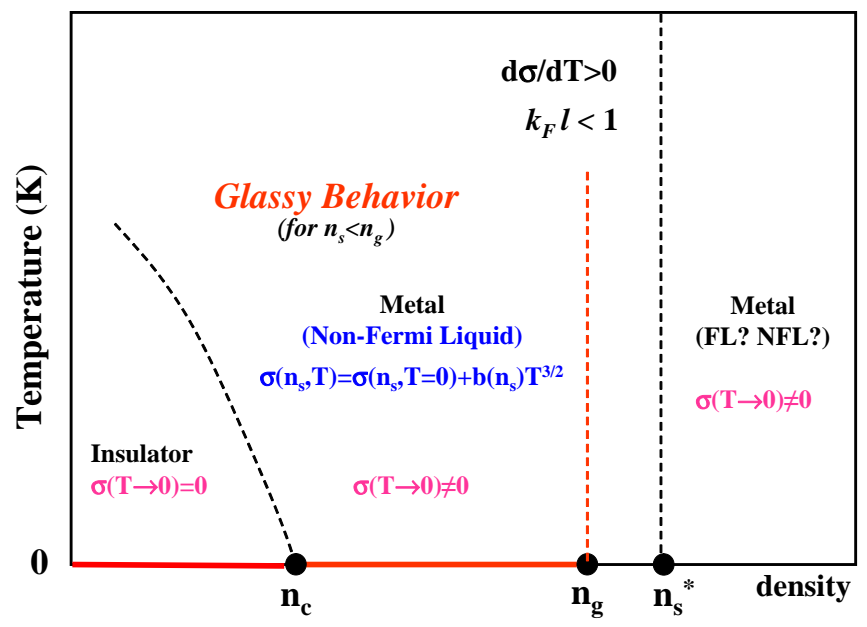

Fig. 0.15 Experimental phase diagram of the 2DES in Si MOSFETs. The metal-insulator transition takes place at the density $n_{c}$ and at $T=0$ in all samples regardless of the amount of disorder. The nature of the metallic phase at high $n_{s}$, such that $k_{F} l>1$, is still under debate [see Kravchenko and Sarachik (2004)]. The glass transition takes place at $T_{g}=0$ for all $n_{s}<n_{g}$. In general, the glass transition is a precursor to the metal-insulator transition, i.e. $n_{c}<n_{g}$, giving rise to an intermediate metallic phase with a particular form of the non-Fermi liquid temperature dependence of conductivity. The aging properties of the glass change abruptly at $n_{c}$, indicating different natures of the insulating and metallic glass phases. For sufficiently low disorder, the intermediate phase vanishes: $n_{c} \lesssim n_{s}^{*} \approx n_{g}$.

natures of the insulating glass and metallic glass phases are different. The 2DES in Si thus exhibits all the characteristics common to other out-of-equilibrium systems, regardless of their dimensionality. Numerous similarities to spin glasses with longrange RKKY interaction are particularly remarkable. Here, however, only long-range Coulomb interaction and potential scattering are present, and measurements in a parallel magnetic field confirm that charge degrees of freedom, not spin, are responsible for glassy freezing. Therefore, the experiments show that the 2D MIT is closely related to the melting of this Coulomb glass.

\subsection{Discussion}

Experiments on a 2DES in Si clearly provide strong support to theoretical proposals describing the 2D MIT as the melting of a Coulomb glass (Dobrosavljević et al., 2003 Thakur and Neilson, 1996: Thakur and Neilson, 1999: Chakravarty et al., 1999: Pastor and Dobrosavljević, 1999 Dalidovich and Dobrosavljević, 2002). In particular, the model of the MIT as a Mott transition with disorder (Dobrosavljević et al., 2003) predicts the emergence of an intermediate metallic glass phase in sufficiently disordered systems (Fig. 0.16). Changing 


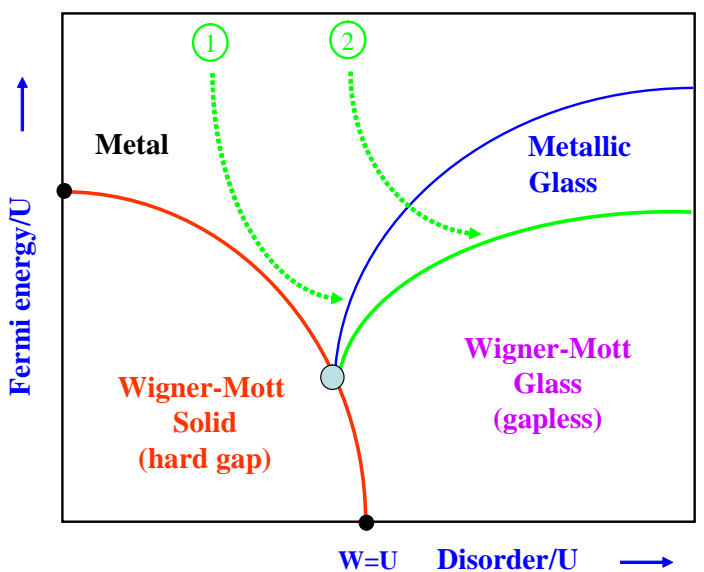

Fig. 0.16 Theoretical phase diagram (adapted from Dobrosavljević et al. 2003). The Fermi energy and disorder on the two axes are expressed in units of the on-site interaction $U$. For large enough disorder, the metal-insulator transition is preceded by the glass transition, giving rise to an intermediate, metallic glass phase. Experimental trajectories, where the MIT is approached by varying $n_{s}$, are represented schematically by the green curved arrows for the cases of low (trajectory 1) and high (trajectory 2) disorder.

the carrier density in highly disordered samples, for example, corresponds te 10 trajectory 2 in the theoretical phase diagram in Fig. 0.16, where the intermediate phase is predicted to be relatively broad. On the other hand, for low-disordered samples (trajectory 1 ), the metallic glass phase is very narrow or it vanishes, in agreement with experimental observations. The same theory predicts (Dalidovich and Dobrosavljević, 2002) the $T^{3 / 2}$ correction to conductivity in the metallic glass phase, which is precisely what is found in the experiment. The emergence of the metallic glass phase, its dependence on the disorder, and the specific form of $\sigma(T)$ in this regime were obtained using a mean-field theory approach, which is known to produce hierarchical dynamics and aging in models of other glasses. Therefore, this approach seems promising in describing also the glassy charge dynamics in a $2 \mathrm{DES}$ in Si. There is currently no other theory available that predicts any of these experimental features.

A molecular dynamics simulation of the crossover from a Wigner liquid to a Wigner glass in a 2D system of interacting electrons with disorder (Reichhardt and Reichhardt, 2004) has reproduced the main noise results (Sec. 0.3.1). In particular, a strong, orders of magnitude increase in the noise power and a jump in the exponent $\alpha$ was found at low $T$ and $n_{s}$, as well as the emergence of non-Gaussianity. By looking at the electron trajectories for a fixed period of time (Fig. 0.17), it was found that, at the highest $n_{s}$, electrons can flow freely throughout the sample. As $n_{s}$ is reduced, electron motion consists of a mixture of $2 \mathrm{D}$ and $1 \mathrm{D}$ regions (Fig. 0.17 upper right), but over longer times,

\footnotetext{
${ }^{10}$ Since $E_{F} \sim n_{s}$ and $U \sim n_{s}^{1 / 2}$ in $2 \mathrm{D}$ and the disorder $W \approx$ const, the change of $n_{s}$ in the experiment is described by $\left(E_{F} / U\right) \sim(W / U)^{-1}$ in the phase diagram.
} 

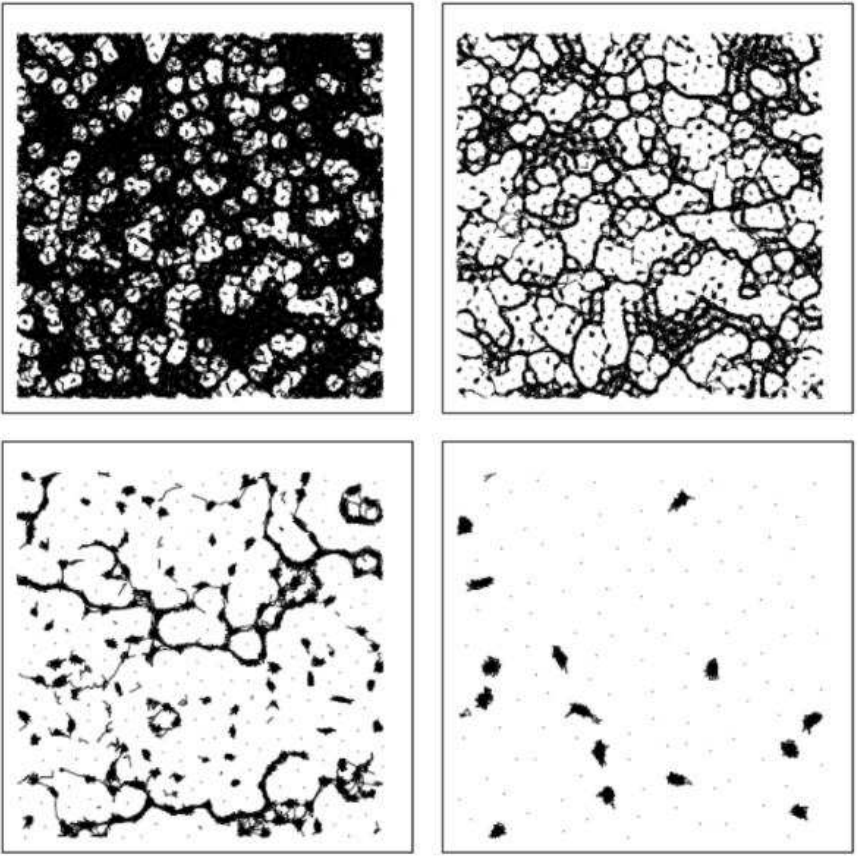

Fig. 0.17 Electron trajectories for a fixed period of time for a fixed $T$ and four electron densities, with the highest $n_{1}$ at upper left, $n_{2}<n_{1}$ upper right, $n_{3}<n_{2}$ lower left, and the lowest $n_{4}<n_{3}$ lower right. Adapted from Ref. (Reichhardt and Reichhardt, 2004).

the motion still occurs throughout the whole sample. At even lower $n_{s}$ (Fig. 0.17lower left), motion occurs mostly through 1D channels that percolate through the sample. Importantly, the channel structures change very slowly with time: some channels close and others open up. Since transport is dominated by a small number of channels, this give rise to large fluctuations in the conductivity and strong correlations. Therefore, in this model, the large noise is due to dynamical inhomogeneities, similar to observations in other glasses (Glotzer, 2000; Richert, 2002). At the lowest $n_{s}$, the channels disappear and transport occurs via jumps between localized regions. This corresponds to the deep insulating regime. In addition, a completely different technique, namely, a Monte Carlo simulation of a 3D Coulomb glass out of equilibrium has also found evidence for similar dynamical heterogeneities (Kolton et al., 2005).

There have been few experimental attempts so far to probe the charge dynamics near the MIT in other materials. The most exciting and important has been the study of the resistance noise in bulk doped Si (Kar et al., 2003), which has been a prototypical system for investigating the critical behavior near the MIT (Miranda and Dobrosavljević, 2005 Sarachik, 1995) for several decades. The results are strikingly similar to those on a 2DES in Si, namely: $i$ ) the noise power increases by several orders of magnitude at $n_{c}$ [Fig. $0.18(\mathrm{a})]$, ii) as $T$ decreases, the noise magnitude increases essentially exponentially for dopings $n / n_{c} \leq 1$ (Fig. 0.18(a) right inset), iii) near the MIT, the exponent 

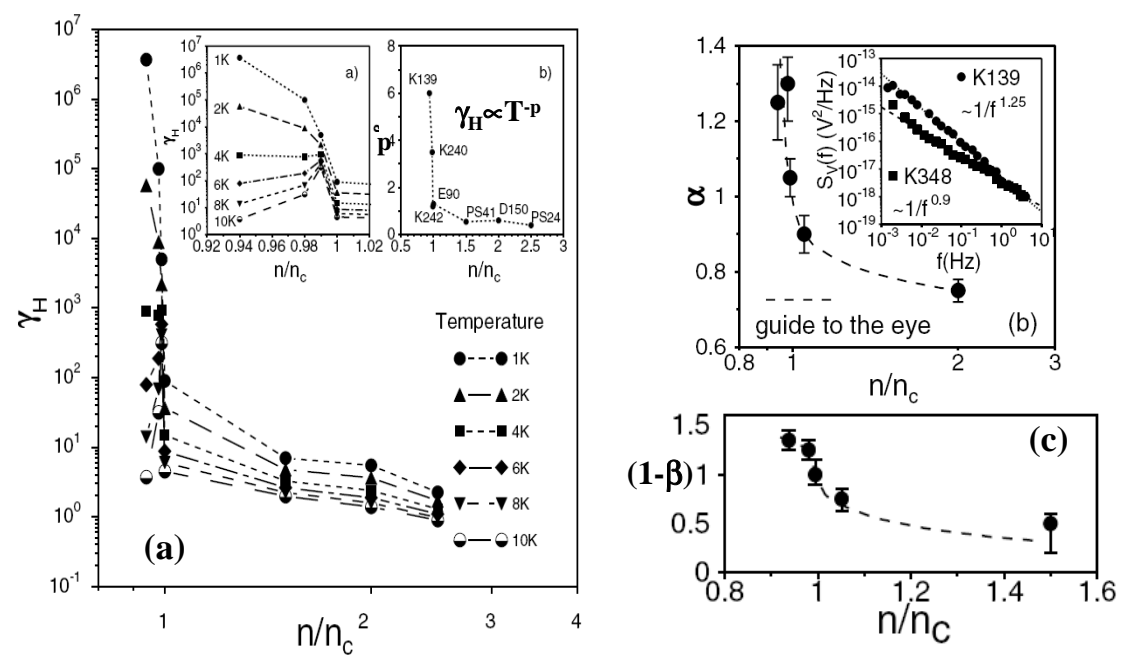

Fig. 0.18 Resistance noise in P-doped Si. Adapted from Kar et al. (2003). (a) Noise magnitude as a function of doping at different $T$. Left inset: the same graph, magnified around $n_{c}$. Right inset: the temperature dependence of the noise magnitude vs. doping. (b) Exponent $\alpha$ vs. doping. Inset: the power spectrum $S(f) \propto 1 / f^{\alpha}$. (c) Exponent $(1-\beta)$, a measure of correlations, vs. doping.

$\alpha$ rapidly increases to a value much larger than 1 [Fig. 0.18(b)], and $i v)$ the exponent $(1-\beta)$ of the second spectrum becomes strongly nonwhite $(\neq 0)$ near $n_{c}$ [Fig. [0.18(c)], indicating the onset of correlated dynamics. The similarity of the non-Gaussian spectra observed in both 2DES and in 3D doped Si near the MIT supports the intriguing possibility that such correlated dynamics may indeed be a universal feature of the MIT regardless of the dimensionality.

Resistance noise was also measured in very clean 2D hole systems (2DHS) in GaAs (Leturcq et al., 2003: Deville et al., 2005: Deville et al., 2006). Even though an orders of magnitude enhancement of the noise was observed with decreasing $T$ and hole density $p_{s}$, similar to the results on 2DES in Si and bulk doped Si, no evidence for strong correlations or glassiness was found. It is likely that the (lack of) disorder here plays a crucial role, since glassiness is not expected theoretically in sufficiently clean systems (Dobrosavljević et al., 2003) (Fig. 0.16). It is interesting, though, that the behavior of transport and noise in an intermediate regime of $p_{s}$ was attributed to the coexistence of nanoscale conducting and insulating regions, consistent with other studies that favor the percolation picture in 2DHS and 2DES in GaAs (Ilani et al., 2000 Ilani et al., 2001; Gao et al., 2002; Allison et al., 2006). The local probes provide evidence (Ilani et al., 2000, Ilani et al., 2001) that as the density approaches the critical hole density $p_{c}$ from the metallic side, the 2DHS fragments into localized charge configurations that are distributed in space, so that the insulating phase is spatially inhomogeneous. The complicated structure emerges already on the metallic side of the 
MIT, reminiscent of the onset of glassiness in disordered 2DES in Si. Clearly, more work is needed to examine the charge dynamics in these systems, especially in the presence of a higher amount of disorder.

The resistance noise techniques, long used in studies of various glassy systems, are starting to be recognized as powerful tools also in the investigations of the dynamics of cuprates (Raičević et al., 2007; Raičević et al., 2008; Raičević et al., 2011 . Bonetti et al., 2004 Fruchter et al., 2007, Caplan et al., 2010). The currently available data, however, are scarce and difficult to compare because they probe very different materials and regions of phase space. This warrants further systematic studies of the charge dynamics in various cuprates as a function of all the relevant parameters, such as doping and disorder, as well as different growth techniques. Comparative studies of charge dynamics in a 2DES in $\mathrm{Si}$, as an excellent model system for the MIT and out-of-equilibrium behavior, and in more complex materials, such as cuprates, should provide important information on these two fundamental problems of condensed matter physics.

\subsection{Acknowledgments}

This work was supported by NSF Grant DMR-0905843 and the National High Magnetic Field Laboratory via NSF Grant DMR-0654118. 


\section{References}

Alba, M., Hammann, J., Ocio, M., Refregier, Ph., and Bouchiat, H. (1987). Spinglass dynamics from magnetic noise, relaxation, and susceptibility measurements. J. Appl. Phys., 61, 3683.

Alba, M., Ocio, M., and Hammann, J. (1986). Ageing process and response function in spin glasses: An analysis of the thermoremanent magnetization decay in Ag: $\mathrm{Mn}$ (2.6\%). Europhys. Lett., 2, 45.

Allison, G. D., Galaktionov, E. A., Savchenko, A. K., Safonov, S. S., Fogler, M. M., Simmons, M. Y., and Ritchie, D. A. (2006). Thermodynamic density of states of two-dimensional GaAs systems near the apparent metal-insulator transition. Phys. Rev. Lett., 96, 216407.

Altshuler, B. L., Maslov, D. L., and Pudalov, V. M. (2001). Metal-insulator transition in 2D: resistance in the critical region. Physica (Amsterdam), 9E, 209.

Amir, A., Oreg, Y., and Imry, Y. (2011). Electron glass dynamics. Annu. Rev. Condens. Matter Phys., 2, 235.

Ando, T., Fowler, A. B., and Stern, F. (1982). Electronic properties of twodimensional systems. Rev. Mod. Phys., 54, 437.

Barrat, J.-L., Feigelman, M., Kurchan, J., and Dalibard, J. (ed.) (2003). Slow Relaxations and Nonequilibrium Dynamics in Condensed Matter. Springer, Berlin.

Belitz, D. and Kirkpatrick, T. R. (1994). The Anderson-Mott transition. Rev. Mod. Phys., 66, 261.

Belitz, D. and Kirkpatrick, T. R. (1995). Order parameter description of the Anderson-Mott transition. Z. Phys. B, 98, 513.

Ben-Chorin, M., Ovadyahu, Z., and Pollak, M. (1993). Nonequilibrium transport and slow relaxation in hopping conductivity. Phys. Rev. B, 48, 15025.

Bielejec, E. and Wu, W. (2001). Electron glass in ultrathin granular Al films at low temperatures. Phys. Rev. Lett., 87, 256601.

Binder, K. and Young, A. P. (1986). Spin glasses: Experimental facts, theoretical concepts, and open questions. Rev. Mod. Phys., 58, 801.

Bogdanovich, S., Dai, P., Sarachik, M. P., Dobrosavljević, V., and Kotliar, G. (1997). Scaling of the conductivity of Si:B: Anomalous crossover in a magnetic field. Phys. Rev. B, 55, 4215 .

Bogdanovich, S. and Popović, D. (2002a). Glass transition in a two-dimensional electron system in silicon. Physica E, 12, 604.

Bogdanovich, S. and Popović, D. (2002b). Onset of glassy dynamics in a twodimensional electron system in silicon. Phys. Rev. Lett., 88, 236401. Erratum, Phys. Rev. Lett. 89, 289904 (2002).

Bonetti, J. A., Caplan, D. S., Harlingen, D. J. Van, and Weissman, M. B. (2004). Electronic transport in underdoped $\mathrm{YBa}_{2} \mathrm{Cu}_{3} \mathrm{O}_{7-\delta}$ nanowires: evidence for fluctu- 
ating domain structures. Phys. Rev. Lett., 93, 087002.

Bouchaud, J.-P., Cugliandolo, L. F., Kurchan, J., and Mezard, M. (1997). Out of equilibrium dynamics in spin-glasses and other glassy systems. In Spin Glasses and Random Fields (ed. A. P. Young). World Scientific, Singapore.

Camjayi, A., Haule, K., Dobrosavljević, V., and Kotliar, G. (2008). Coulomb correlations and the Wigner-Mott transition. Nature Phys., 4, 932.

Caplan, D. S., Orlyanchik, V., Weissman, M. B., Harlingen, D. J. Van, Fradkin, E. H., Hinton, M. J., and Lemberger, T. R. (2010). Anomalous noise in the pseudogap regime of $\mathrm{YBa}_{2} \mathrm{Cu}_{3} \mathrm{O}_{7-\delta}$. Phys. Rev. Lett., 104, 177001.

Castellani, C., Kotliar, G., and Lee, P. A. (1987). Fermi-liquid theory of interacting disordered systems and the scaling theory of the metal-insulator transition. Phys. Rev. Lett., 59, 323.

Chakravarty, S., Kivelson, S., Nayak, C., and Voelker, K. (1999). Wigner glass, spin liquids and the metal-insulator transition. Philos. Mag. B, 79, 859.

Committee on CMMP 2010, National Research Council (2007). Condensed-Matter and Materials Physics: The Science of the World Around Us. The National Academies Press, Washington, D.C.

Dagotto, E. (2002). Nanoscale phase separation and colossal magnetoresistance. Springer-Verlag, Berlin.

Dagotto, E. (2005). Complexity in strongly correlated electronic systems. Science, 309, 257-262.

Dalidovich, D. and Dobrosavljević, V. (2002). Landau theory of the Fermi-liquid to electron-glass transition. Phys. Rev. B, 66, 081107.

Davies, J. H., Lee, P. A., and Rice, T. M. (1982). Electron glass. Phys. Rev. Lett., 49, 758 .

Davies, J. H., Lee, P. A., and Rice, T. M. (1984). Properties of the electron glass. Phys. Rev. B, 29, 4260.

Deville, G., Leturcq, R., L'Hôte, D., Tourbot, R., Mellor, C. J., and Henini, M. (2005). $1 / f$ noise in low density two-dimensional hole systems in GaAs. AIP Conf. Proc., 780, 139.

Deville, G., Leturcq, R., L'Hôte, D., Tourbot, R., Mellor, C. J., and Henini, M. (2006). $1 / f$ noise in a dilute GaAs two-dimensional hole system in the insulating phase. Physica E, 34, 252.

Dobrosavljević, V., Tanasković, D., and Pastor, A. A. (2003). Glassy behavior of electrons near metal-insulator transitions. Phys. Rev. Lett., 90, 016402.

Dolgopolov, V. T. and Gold, A. (2000). Magnetoresistance of a two-dimensional electron gas in a parallel magnetic field. JETP Lett., 71, 27.

Emery, V. J. and Kivelson, S. A. (1995). Superconductivity in bad metals. Phys. Rev. Lett., 74, 3253.

Eng, K., Feng, X. G., Popović, D., and Washburn, S. (2001). Effects of a parallel magnetic field on the novel metallic behavior in two dimensions. Springer Proceedings in Physics, 87, 741.

Eng, K., Feng, X. G., Popović, D., and Washburn, S. (2002). Effects of a parallel magnetic field on the metal-insulator transition in a dilute two-dimensional electron system. Phys. Rev. Lett., 88, 136402. 
Feng, X. G., Popović, D., and Washburn, S. (1999). Effect of local magnetic moments on the metallic behavior in two dimensions. Phys. Rev. Lett., 83, 368.

Feng, X. G., Popović, D., Washburn, S., and Dobrosavljević, V. (2001). Novel metallic behavior in two dimensions. Phys. Rev. Lett., 86, 2625.

Fletcher, R., Pudalov, V. M., Radcliffe, A. D. B., and Possanzini, C. (2001). Critical behaviour of thermopower and conductivity at the metal-insulator transition in high-mobility Si-MOSFETs. Semicond. Sci. Tech., 16, 386.

Fruchter, L., Raffy, H., and Li, Z. Z. (2007). Resistance noise in $\mathrm{Bi}_{2} \mathrm{Sr}_{2} \mathrm{CaCu}_{2} \mathrm{O}_{8+\delta}$. Phys. Rev. B, 76, 212503.

Gao, X. P. A., Mills, A. P., Ramirez, A. P., Pfeiffer, L. N., and West, K. W. (2002). Weak-localization-like temperature-dependent conductivity of a dilute twodimensional hole gas in a parallel magnetic field. Phys. Rev. Lett., 89, 16801.

Glotzer, S. C. (2000). Spatially heterogeneous dynamics in liquids: insights from simulation. J. Non-Cryst. Solids, 274, 342.

Goldenfeld, N. (1992). Lectures on Phase Transitions and the Renormalization Group. Addison-Wesley.

Gor'kov, L. P. and Sokol, A. V. (1987). Localized and delocalized states and the properties of the normal phase of recently discovered superconductors. JETP Lett., 46, 420 .

Grempel, D. R. (2004). Off-equilibrium dynamics of the two-dimensional Coulomb glass. Europhys. Lett., 66, 854.

Grenet, T. (2003). Symmetrical field effect and slow electron relaxation in granular aluminium. Eur. Phys. J. B, 32, 275.

Grenet, T., Delahaye, J., Sabra, M., and Gay, F. (2007). Anomalous electric-field effect and glassy behaviour in granular aluminium thin films: electron glass? Eur. Phys. J. B, 56, 183.

Grünewald, M., Pohlman, B., Schweitzer, L., and Würtz, D. (1982). Mean field approach to the electron glass. J. Phys. C, 15, L1153.

Herbut, I. F. (2001). The effect of parallel magnetic field on the Boltzmann conductivity and the Hall coefficient of a disordered two-dimensional Fermi liquid. Phys. Rev. B, 63, 113102 .

Hernandez, L. M., Bhattacharya, A., Parendo, K. A., and Goldman, A. M. (2003). Electrical transport of spin-polarized carriers in disordered ultrathin films. Phys. Rev. Lett., 91, 126801.

Hewson, A. C. (1993). The Kondo Problem to Heavy Fermions. Cambridge Univ. Press, Cambridge, England.

Hodge, I. M. (1995). Physical aging in polymer glasses. Science, 267, 1945.

Hohenberg, P. C. and Halperin, B. I. (1977). Theory of dynamic critical phenomena. Rev. Mod. Phys., 49, 435.

Hooge, F. N. (1976). 1/f noise. Physica (Amsterdam), 83B, 14.

Ilani, S., Yacoby, A., Mahalu, D., and Shtrikman, H. (2000). Unexpected behavior of the local compressibility near the $B=0$ metal-insulator transition. Phys. Rev. Lett., 84, 3133.

Ilani, S., Yacoby, A., Mahalu, D., and Shtrikman, H. (2001). Microscopic structure of the metal-insulator transition in two dimensions. Science, 292, 1354. 
Jaroszyński, J. and Popović, D. (2006). Nonexponential relaxations in a twodimensional electron system in silicon. Phys. Rev. Lett., 96, 037403.

Jaroszyński, J. and Popović, D. (2007a). Aging effects across the metal-insulator transition in two dimensions. Phys. Rev. Lett., 99, 216401.

Jaroszyński, J. and Popović, D. (2007b). Nonequilibrium relaxations and aging effects in a two-dimensional Coulomb glass. Phys. Rev. Lett., 99, 046405.

Jaroszyński, J. and Popović, D. (2009). Aging and memory in a two-dimensional electron system in Si. Physica B, 404, 466.

Jaroszyński, J., Popović, D., and Klapwijk, T. M. (2002a). Low-frequency resistance noise studies across the metal-insulator transition in silicon MOSFETs. Physica $E, \mathbf{1 2}, 612$.

Jaroszyński, J., Popović, D., and Klapwijk, T. M. (2002b). Universal behavior of the resistance noise across the metal-insulator transition in silicon inversion layers. Phys. Rev. Lett., 89, 276401.

Jaroszyński, J., Popović, D., and Klapwijk, T. M. (2004a). Glassy behavior of a two-dimensional electron system in $\mathrm{Si}$ in parallel magnetic fields. Proceedings of SPIE, 5469, 95.

Jaroszyński, J., Popović, D., and Klapwijk, T. M. (2004b). Magnetic field dependence of the anomalous noise behavior in a two-dimensional electron system in silicon. Phys. Rev. Lett., 92, 226403.

Jaroszyński, J., Wróbel, J., G.Karczewski, Wojtowicz, T., and Dietl, T. (1998). Magnetoconductance noise and irreversibilities in submicron wires of spin-glass $n^{+}$$\mathrm{Cd}_{1-x} \mathrm{Mn}_{x}$ Te. Phys. Rev. Lett., 80, 5635 .

Jelbert, G. R., Sasagawa, T., Fletcher, J. D., Park, T., Thompson, J. D., and Panagopoulos, C. (2008). Measurement of low energy charge correlations in underdoped spin-glass La-based cuprates using impedance spectroscopy. Phys. Rev. $B, \mathbf{7 8}, 132513$.

Jönsson, P. E. and Takayama, H. (2005). "Glassy dynamics" in Ising spin glasses experiment and simulation. J. Phys. Soc. Jpn, 74, 1131.

Jonsson, T., Jonason, K., and Nordblad, P. (1999). Relaxation of the field-cooled magnetization of an Ising spin glass. Phys. Rev. B, 59, 9402.

Kar, S., Raychaudhuri, A. K., Ghosh, A., v. Löhneysen, H., and Weiss, G. (2003). Observation of non-Gaussian conductance fluctuations at low temperatures in $\mathrm{Si}: \mathrm{P}(\mathrm{B})$ at the metal-insulator transition. Phys. Rev. Lett., 91, 216603.

Kim, N.-J., Popović, D., and Washburn, S. (1998). Phase diagram and validity of one-parameter scaling near the two-dimensional metal-insulator transition. condmat/9809357.

Kirkpatrick, T. R. and Belitz, D. (1994). Anderson-Mott transition as a random-field problem. Phys. Rev. Lett., 74, 1178.

Kivelson, S. A., Bindloss, I. P., Fradkin, E., Oganesyan, V., Tranquada, J. M., Kapitulnik, A., and Howald, C. (2003). How to detect fluctuating stripes in the hightemperature superconductors. Rev. Mod. Phys., 75, 1201.

Kohsaka, Y., Taylor, C., Fujita, K., Schmidt, A., Lupien, C., Hanaguri, T., Azuma, M., Takano, M., Eisaki, H., Takagi, H., Uchida, S., and Davis, J. C. (2007). An intrinsic bond-centered electronic glass with unidirectional domains in underdoped 
cuprates. Science, 315, 1380.

Kolton, A. B., Grempel, D. R., and Domínguez, D. (2005). Heterogeneous dynamics of the three-dimensional Coulomb glass out of equilibrium. Phys. Rev. B, 71, 024206.

Kravchenko, S. V., Kravchenko, G. V., Furneaux, J. E., Pudalov, V. M., and D'Iorio, M. (1994). Possible metal-insulator transition at $B=0$ in two dimensions. Phys. Rev. B, 50, 8039 .

Kravchenko, S. V., Mason, W. E., Bowker, G. E., Furneaux, J. E., Pudalov, V. M., and D'Iorio, M. (1995). Scaling of an anomalous metal-insulator transition in a two-dimensional system in silicon at $B=0$. Phys. Rev. B, 51, 7038.

Kravchenko, S. V. and Sarachik, M. P. (2004). Metal-insulator transition in twodimensional electron systems. Rep. Prog. Phys., 67, 1.

Kurzweil, N. and Frydman, A. (2007). Inverse slow relaxation in granular hopping systems. Phys. Rev. B, 75, 020202(R).

Lebanon, E. and Müller, M. (2005). Memory effect in electron glasses: theoretical analysis via a percolation approach. Phys. Rev. B, 72, 174202.

Lee, M., Oikonomou, P., Segalova, P., Rosenbaum, T. F., Hoekstra, A. F. Th., and Littlewood, P. B. (2005). The electron glass in a switchable mirror: relaxation, ageing and universality. J. Phys. Condens. Matter, 17, L439.

Lee, P. A. and Ramakrishnan, T. V. (1985). Disordered electronic systems. Rev. Mod. Phys., 57, 287.

Leturcq, R., L'Hôte, D., Tourbot, R., Mellor, C. J., and Henini, M. (2003). Resistance noise scaling in a dilute two-dimensional hole system in GaAs. Phys. Rev. Lett., 90, 076402 .

Leuzzi, L. and Nieuwenhuizen, T. M. (2008). Thermodynamics of the Glassy State. Taylor \& Francis, New York.

Levy, P., Parisi, F., Granja, L., Indelicato, E., and Polla, G. (2002). Novel dynamical effects and persistent memory in phase separated manganites. Phys. Rev. Lett., 89, 137001 .

Martinez-Arizala, G., Christiansen, C., Grupp, D. E., Markovic, N., Mack, A. M., and Goldman, A. M. (1998). Coulomb-glass-like behavior of ultrathin films of metals. Phys. Rev. B, 57, R670.

Martinez-Arizala, G., Grupp, D. E., Christiansen, C., Mack, A. M., Markovic, N., Seguchi, Y., and Goldman, A. M. (1997). Anomalous field effect in ultrathin films of metals near the superconductor-insulator transition. Phys. Rev. Lett., 78, 1130.

Miranda, E. and Dobrosavljević, V. (2005). Disorder-driven non-Fermi liquid behaviour of correlated electrons. Rep. Prog. Phys., 68, 2337.

Miyashita, S., Tanaka, S., and Hirano, M. (2007). Nonmonotonic relaxation in systems with reentrant-type interaction. J. Phys. Soc. Jpn, 76, 083001.

Monroe, D. (1990). Capacitance measurements of the dynamics of screening in the electron glass. In Hopping and Related Phenomena 5 (ed. H. Fritzsche and M. Pollak). World Scientific, Singapore.

Monroe, D., Gossard, A. C., English, J. H., Golding, B., Haemmerle, W. H., and Kastner, M. A. (1987). Long-lived Coulomb gap in a compensated semiconductor the electron glass. Phys. Rev. Lett., 59, 1148.

Morita, H. and Kaneko, K. (2005). Roundabout relaxation: collective excitation 
requires a detour to equilibrium. Phys. Rev. Lett., 94, 087203.

Müller, M. and Ioffe, L. B. (2004). Glass transition and the Coulomb gap in electron glasses. Phys. Rev. Lett., 93, 256403.

Müller, M. and Lebanon, E. (2005). History dependence, memory and metastability in electron glasses. J. Phys. IV France, 131, 167.

Nelson, P. (2003). Biological physics: energy, information, life, Chapter 12. W. H. Freeman \& Co., New York.

Neuttiens, G., Strunk, C., Haesendonck, C. V., and Bruynseraede, Y. (2000). Universal conductance fluctuations and low-temperature $1 / f$ noise in mesoscopic AuFe spin glasses. Phys. Rev. B, 62, 3905.

Ogielski, A. T. (1985). Dynamics of three-dimensional Ising spin glasses in thermal equilibrium. Phys. Rev. B, 32, 7384.

Okamoto, T., Hosoya, K., Kawaji, S., and Yagi, A. (1999). Spin degree of freedom in a two-dimensional electron liquid. Phys. Rev. Lett., 82, 3875.

Orenstein, J. and Millis, A. J. (2000). Advances in the physics of high-temperature superconductivity. Science, $\mathbf{2 8 8}, 468$.

Orlyanchik, V. and Ovadyahu, Z. (2004). Stress aging in the electron glass. Phys. Rev. Lett., 92, 066801.

Ovadyahu, Z. (2006a). Quench-cooling procedure compared with the gate protocol for aging experiments in electron glasses. Phys. Rev. B, 73, 214204.

Ovadyahu, Z. (2006b). Temperature- and field-dependence of dynamics in electron glasses. Phys. Rev. B, 73, 214208.

Ovadyahu, Z. and Pollak, M. (1997). Disorder and magnetic field dependence of slow electronic relaxation. Phys. Rev. Lett., 79, 459.

Paalanen, M. A., Rosenbaum, T. F., Thomas, G. A., and Bhatt, R. N. (1983). Critical scaling of the conductance in a disordered insulator. Phys. Rev. Lett., 51, 1896.

Pankov, S. and Dobrosavljević, V. (2005). Nonlinear screening theory of the Coulomb glass. Phys. Rev. Lett., 94, 046402.

Pappas, C., Mezei, F., Ehlers, G., Manuel, P., and Campbell, I. A. (2003). Dynamic scaling in spin glasses. Phys. Rev. B, 68, 054431.

Pastor, A. A. and Dobrosavljević, V. (1999). Melting of the electron glass. Phys. Rev. Lett., 83, 4642.

Pollak, M. (1984). Non-ergodic behaviour of Anderson insulators with and without Coulomb interactions. Philos. Mag. B, 50, 265.

Pollak, M. and Ortuño, M. (1982). Coulomb interactions in Anderson localized disordered systems. Sol. Energy Mater., 8, 81.

Popović, D., Bogdanovich, S., Jaroszyński, J., and Klapwijk, T. M. (2003). Metalinsulator transition and glassy behavior in two-dimensional electron systems. Proceedings of SPIE, 5112, 99.

Popović, D., Fowler, A. B., and Washburn, S. (1997). Metal-insulator transition in two dimensions: effects of disorder and magnetic field. Phys. Rev. Lett., 79, 1543.

Pudalov, V. M., Brunthaler, G., Prinz, A., and Bauer, G. (1998a). Lack of universal one-parameter scaling in the two-dimensional metallic regime. JETP Lett., 68, 442. Pudalov, V. M., Brunthaler, G., Prinz, A., and Bauer, G. (1998b). Logarithmic temperature dependence of the conductivity of the two-dimensional metal. JETP 
Lett., 68, 534 .

Pudalov, V. M., D’Iorio, M., Kravchenko, S. V., and Campbell, J. W. (1993). Zeromagnetic-field collective insulator phase in a dilute 2D electron system. Phys. Rev. Lett., 70, 1866.

Raičević, I., Jaroszyński, J., Popović, D., Jelbert, G., Panagopoulos, C., and Sasagawa, T. (2007). Low-temperature resistance noise in lightly doped $\mathrm{La}_{2-x} \mathrm{Sr}_{x} \mathrm{CuO}_{4}$. Proceedings of SPIE, 6600, 660020.

Raičević, I., Jaroszyński, J., Popović, D., Panagopoulos, C., and Sasagawa, T. (2008). Evidence for charge glasslike behavior in lightly doped $\mathrm{La}_{2-x} \mathrm{Sr}_{x} \mathrm{CuO}_{4}$ at low temperatures. Phys. Rev. Lett., 101, 177004.

Raičević, I., Popović, D., Panagopoulos, C., and Sasagawa, T. (2011). Non-Gaussian noise in the in-plane transport of lightly doped $\mathrm{La}_{2-x} \mathrm{Sr}_{x} \mathrm{CuO}_{4}$ : Evidence for a collective state of charge clusters. Phys. Rev. B, 83, 195133.

Reichhardt, C. and Reichhardt, C. J. Olson (2004). Noise at the crossover from Wigner liquid to Wigner glass. Phys. Rev. Lett., 93, 176405.

Richert, R. (2002). Heterogeneous dynamics in liquids: fluctuations in space and time. J. Phys.: Condens. Matter, 14, R703.

Rodriguez, G. F., Kenning, G. G., and Orbach, R. (2003). Full aging in spin glasses. Phys. Rev. Lett., 91, 037203.

Rosenbaum, T. F., Andres, K., Thomas, G. A., and Bhatt, R. N. (1980). Sharp metal-insulator transition in a random solid. Phys. Rev. Lett., 45, 1723.

Rosenbaum, T. F., Field, S. B., and Bhatt, R. N. (1989). Variation of the metallic onset with magnetic field in doped germanium. Europhys. Lett., 10, 269.

Rubi, M. and Perez-Vicente, C. (ed.) (1997). Complex behavior of glassy systems. Volume 492, Lecture Notes in Physics. Springer, Berlin.

Sachdev, S. (1999). Quantum Phase Transitions. Cambridge University Press, UK. Sarachik, M. P. (1995). Transport studies in doped semiconductors near the metalinsulator transition. In The Metal-Nonmetal Transition Revisited: A Tribute to Sir Nevill Mott (ed. P. P. Edwards and C. N. Rao). Francis and Taylor Ltd.

Sarachik, M. P., Simonian, D., Kravchenko, S. V., Bogdanovich, S., Dobrosavljević, V., and Kotliar, G. (1998). Metal-insulator transition in Si:X (X=P,B): Anomalous response to a magnetic field. Phys. Rev. B, 58, 6692.

Schmalian, J. and Wolynes, P. G. (2000). Stripe glasses: Self-generated randomness in a uniformly frustrated system. Phys. Rev. Lett., 85, 836.

Scofield, J. H. (1987). ac method for measuring low-frequency resistance fluctuation spectra. Rev. Sci. Instrum., 58, 985-993.

Shashkin, A. A., Kravchenko, S. V., and Klapwijk, T. M. (2001). Metal-insulator transition in a 2D electron gas: Equivalence of two approaches for determining the critical point. Phys. Rev. Lett., 87, 266402.

Sjöstrand, M. E., Cole, T., and Stiles, P. J. (1976). Low temperature saturation of the channel conductivity in silicon inversion layers. Surf. Sci., 58, 72.

Sjöstrand, M. E. and Stiles, P. J. (1975). The channel conductivity in n-type Si inversion layers at very low electron densities. Solid State Commun., 16, 903.

Struik, L. C. E. (1978). Physical aging in amorphous polymers and other materials. Elsevier, Amsterdam. 
Thakur, J. S. and Neilson, D. (1996). Frozen electron solid in the presence of small concentrations of defects. Phys. Rev. B, 54, 7674 .

Thakur, J. S. and Neilson, D. (1999). Phase diagram of the metal-insulator transition in two-dimensional electronic systems. Phys. Rev. B, 59, R5280.

Thorsmølle, V. K. and Armitage, N. P. (2010). Ultrafast (but many-body) relaxation in a low-density electron glass. Phys. Rev. Lett., 105, 086601.

Tutuc, E., De Poortere, E. P., Papadakis, S. J., and Shayegan, M. (2001). In-plane magnetic field-induced spin polarization and transition to insulating behavior in two-dimensional hole systems. Phys. Rev. Lett., 86, 2858.

Vaknin, A., Ovadyahu, Z., and Pollak, M. (1998). Evidence for interactions in nonergodic electronic transport. Phys. Rev. Lett., 81, 669.

Vaknin, A., Ovadyahu, Z., and Pollak, M. (2000). Aging effects in an Anderson insulator. Phys. Rev. Lett., 84, 3402.

Vaknin, A., Ovadyahu, Z., and Pollak, M. (2002). Nonequilibrium field effect and memory in the electron glass,. Phys. Rev. B, 65, 134208.

Verbruggen, A. H., Stoll, H., Heeck, K., and Koch, R. H. (1989). A novel technique for measuring resistance fluctuations independently of background noise. Appl. Phys. A, 48, 233-236.

Vincent, E. (2007). Aging, rejuvenation and memory: the example of spin glasses. In Ageing and the Glass Transition (ed. M. Henkel, M. Pleimling, and R. Sanctuary), Volume 716, Lecture Notes in Physics, pp. 7-60. Springer.

Vitkalov, S. A., Sarachik, M. P., and Klapwijk, T. M. (2001). Spin polarization of two-dimensional electrons determined from Shubnikov-de Haas oscillations as a function of angle. Phys. Rev. B, 64, 073101.

Vitkalov, S. A., Zheng, H., Mertes, K. M., and Sarachik, M. P. (2000). Small angle Shubnikov-de Haas measurements in a 2D electron system: the effect of a strong in-plane magnetic field. Phys. Rev. Lett., 85, 2164.

Washburn, S., Kim, N. J., Feng, X. G., and Popović, D. (1999a). Scaling laws, phase diagram, localized magnetic moments and Kondo effect in two-dimensional metals. Ann. Phys. (Leipzig), 8, 569.

Washburn, S., Kim, N.-J., Li, K. P., and Popović, D. (1999b). Scaling and universal behavior near the two-dimensional metal-insulator transition. Mol. Phys. Rept., 24, 150.

Watanabe, M., Itoh, K. M., Ootuka, Y., and Haller, E. E. (1999). Metal-insulator transition of isotopically enriched neutron-transmutation-doped 70Ge:Ga in magnetic fields. Phys. Rev. B, 60, 15817.

Weissman, M. B. (1988). $1 / f$ noise and other slow, nonexponential kinetics in condensed matter. Rev. Mod. Phys., 60, 537.

Weissman, M. B. (1993). What is a spin glass? A glimpse via mesoscopic noise. Rev. Mod. Phys., 65, 829.

Weissman, M. B., Israeloff, N. E., and Alers, G. B. (1992). Spin-glass fluctuation statistics: mesoscopic experiments in CuMn. J. Magn. Magn. Mater., 114, 87.

Wróbel, J., Jaroszyński, J., Dietl, T., Regiński, K., and Bugajski, M. (1998). Conductance noise of submicron wires in the regime of quantum Hall effect. Physica (Amsterdam), 256B-258B, 69. 